\title{
Hexagonal Resonator Loaded Star Shaped Polarization Insensitive Compact Broadband Zero Indexed Nano-Meta Absorber for Optical Region Applications: A Numerical Approach
}

Md Samsuzzaman ( $\square$ sobuz@pstu.ac.bd)

Patuakhali Science and Technology University https://orcid.org/0000-0002-6007-4816

Ismail Hossain

Universiti Kebangsaan Malaysia

Ahasanul Hoque

Universiti Kebangsaan Malaysia

Mohammad Tariqul Islam

Universiti Kebangsaan Malaysia

Ali F. Almutairi

Kuwait University

\section{Nano Express}

Keywords: Hexagonal Resonator Loaded, Star Shaped, Polarization Insensitive, Compact Broadband Metamaterial Absorber, Optical Region Applications.

Posted Date: January 26th, 2021

DOI: https://doi.org/10.21203/rs.3.rs-151084/v1

License: (c) (i) This work is licensed under a Creative Commons Attribution 4.0 International License.

Read Full License 


\section{Hexagonal Resonator Loaded Star Shaped Polarization Insensitive Compact Broadband Zero Indexed Nano-Meta Absorber for Optical Region Applications: A Numerical Approach}

\section{Ismail Hossain", Md Samsuzzaman ${ }^{2, *}$, , Ali F. Almutairi ${ }^{3, b, *}$, Ahasanul Hoque ${ }^{4}$, Mohammad Tariqul}

Islam $^{4, *}, \mathrm{c}$

${ }^{1}$ Space Science Center (ANGKASA), Universiti Kebangsaan Malaysia, 43600 UKM Bangi, Selangor, Malaysia

${ }^{2}$ Faculty of Computer Science and Engineering, Patuakhali Science and Technology University, Bangladesh

${ }^{3}$ Electrical Engineering Department, Kuwait University, 13060, Kuwait City, Kuwait

${ }^{4}$ Dept. of Electrical, Electronic and Systems Engineering, Faculty of Engineering and Built Environment, Universiti Kebangsaan Malaysia, Malaysia

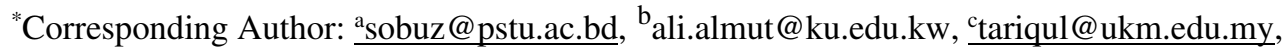




\title{
Hexagonal Resonator Loaded Star Shaped Polarization Insensitive Compact Broadband Zero Indexed Nano-Meta Absorber for Optical Region Applications: A Numerical Approach
}

\begin{abstract}
Broadband response metamaterial absorber (MMA) remains a challenge among researchers. A nanostructured new Zero Indexed Metamaterial (ZIM) absorber is presented in this study, which is constructed with a hexagonal shape resonator for the optical region applications. The design consists of a resonator and dielectric layers made with tungsten and quartz (Fused), respectively. The proposed absorbent exhibits average absorption of more than $0.8972(89.72 \%)$ within the visible wavelength of 450-600 nm and almost perfect absorption $0.99(99 \%)$ at $458.54 \mathrm{~nm}$. Based on computational analysis, the proposed absorber can be characterized as Zero Indexed Metamaterial. The developments of ZIM absorbers have demonstrated plasmonic resonance characteristics and a perfect impedance match. The incidence obliquity in typically the range of $0^{\circ}-90^{\circ}$ both in TE and TM mode with maximum absorbance is more than $0.8972(\sim 89.72 \%)$ and up to $35^{\circ}$ angular stability ,which is suitable for solar cell applications, like exploiting solar energy. The proposed structure prototype is designed and simulated by a study of microwave technology numerical computer simulation (CST) tools. Finite integration technique (FIT) and finite element method (FEM) is performed to data analysis in CST software, and HFSS also helps validate the numerical data of the proposed ZIM absorber. The proposed MMA design is appropriate for substantial amounts of absorption, wide-angle stability, absolute invisible layers, magnetic resonance imaging (MRI), color images, and thermal imaging applications.
\end{abstract}

Keywords: Hexagonal Resonator Loaded; Star Shaped; Polarization Insensitive; Compact Broadband Metamaterial Absorber; Optical Region Applications.

\section{Introduction}

Metamaterial research has already entered an advanced stage where sophisticated electromagnetism, highfrequency materials research with electromagnetism are explored. Veselago explained the dielectric properties of a substance that generally does not match ordinary material; since the values of permittivity and permeability are negative (1). Double negative (DNG) metamaterial was demonstrated for achieving negative permeability and permittivity by varying geometric structures (2). For a substantial variation in metamaterials operating frequency, wavelength free space should remain high in the unit cells (3). This particular characteristic enables metamaterials to be used as antennas (4), waveguides (5), invisibility cloaking (6), absorbers $(7,8)$, and various kinds of sensors (9).

The absorber may be defined as an ideal or close to the absorption unit to absorb the entire incident radiation incident. Absorbers at the specific resonance frequencies depend on the materials. Broadband absorbers in metamaterials are the main causes of incident radiation material properties that are independent of frequency (10). The effective permeability at the negative real part in the MM absorber is explained on electromagnetic wave transmission. Perfect absorbers (PA) are related to the physical and imagined parts of $\varepsilon$ and $\mu$. Electric permittivity and the real part of the specific permeability will be equal for perfect absorbent. The imaginary part should be bigger to repel; usually, the EM wave maximum energy to the PA media (11). Developed the first metamaterial absorber in 2008 and has more than $0.88(88 \%)$ absorbance at $11.5 \mathrm{GHz}$ with FR4 substrate and copper. Furthermore, researchers added that the dielectric constant is consistently incorporated substrate optimizing the small resonant frequency or peak (12). First, metamaterial absorbers at the terahertz frequency region were made and demonstrated by (13). Periodic structure-based metamaterial showed a high absorbance (14). For this reason, as opposed to small thicknesses materials, this material's high frequency could take the form of terahertz to the infrared through a simpler design and less expensive manufacturing process (15). In 2012, the perfect broadband absorber (PBA) introduced and measured $0.995(99.5 \%)$ absorbance at the $13.5 \mathrm{GHz}$ frequency band, where the researchers used FR-4 and copper as the dielectric layer and copper as metal, respectively (11). For the application of detecting and sensing, an air-spacer metamaterial absorber can be used, which is made by using silicon, silicon 
nitride, gold, and polyamide with a complete procedure (9). With a visible wavelength of $400 \mathrm{~nm}$ to $700 \mathrm{~nm}$, almost 0.80 (80\%), the average absorption was identified with the layer concerning $\mathrm{Cu} / \mathrm{Si} 3 \mathrm{~N} 4$ / $\mathrm{Cu}$ on a silicon substrate. At the visible wavelength from $400 \mathrm{~nm}$ to $700 \mathrm{~nm}$, almost $0.80(80 \%)$ average absorption has been found with the $\mathrm{Cu} / \mathrm{Si} 3 \mathrm{~N} 4 / \mathrm{Cu}$ stack coating on a silicon substrate (16). Damper four layers with an arbitrary section near-perfect absorber of the unit have been tested for a wide planar strip MPA, which comprises four peaks and consists of a dielectric place with two gold's (17). Damper dual-band four-layer gold, gallium arsenide (GaAs), and Pyrex (glass) demonstrated 0.9999 (99.99\%) and 0.999 (99.90\%) absorbance (18). The absorber ethanol detection concentration was performed with a three-layer design on 'kodak' premium photo paper, which may shift the resonance frequency of $20 \%$ to $80 \%$ with a linear frequency shifter $10.04 \mathrm{GHz} 8.95 \mathrm{GHz}$. One to three layers using a silicon dioxide thin light absorber $(\mathrm{SiO} 2)$ dividing two metal planes takes greater than $0.90(90 \%)$ of absorbance in visible and infrared regimes (19). Damper three layers using silicon-gold-gold dioxide broadband absorption of $0.90(90 \%)$ in the range of visible wavelengths and infrared regimes, both polarization using a genetic algorithm (GA) (20). Three-layer metamaterial absorber with silver-silicon dioxide-silver had absorbance greater than $0.60(60 \%)$, with a $0.95(95 \%)$ peak value in the optical wavelength (21). A structure comprising two layers of gold $(\mathrm{Au})$ and silicon $(\mathrm{Si})$, having $0.933(93.3 \%)$ above absorptivity in visible ranges complete with $0.991(99.1 \%)$ peak at $453 \mathrm{~nm}$ wavelength (22). A metasurface resonator base absorber with three crossed layers of gold-dielectric glass is above $0.70(70 \%)$ from $400-1000 \mathrm{~nm}$ (23). An ultra-wideband MMA matrix formed of repeated multi-layer metal-dielectric conical logs consisting of seven bimetallic or homogeneous metallic pairs had an associate absorption proficiency of 0.80 (80\%) from 474.4-784.4 nm (24). A thin film resonator having with aluminum (Al) and indium tin oxide (ITO) has a larger specific absorption of $0.90(90 \%)$ for large transmission capacity utilizing $100 \mathrm{~nm}$ P3HT, PCBM and P3HT: PCBM heterostructure (25). One with a peak value of the order of 0.98 (98\%) ultrathin absorber in three layers with a bandwidth of $300 \mathrm{~nm}$ comprising of silver-silver-SiO2 has a greater absorption at $0.80(80 \%)(26)$. As the above discussion shows, broadband perfect absorber (BPA) rarely covers all optical wavelengths with more than $0.90(90 \%)$ absorption at all points. To use BPA in optical wavelength applications is highly required.

The proposed ZIM absorbers incorporates another BPA comprising of metal-dielectric-metal ultrathin nanostructure named metasurface wavelength $450-600 \mathrm{~nm}$. The reviews of the literature show that broadband in MMAS optical regions is not necessarily easily obtainable. Receive tungsten makes a difference in the anticipated design to resist high temperatures and impedance coordinated along with free space for a particular optical range. Quartz (Fused), is also associated with the design's advantage since it has minimal comparative permittivity with lossless properties inside the optical locale and an extraordinary softening stage. This proposed geometrical structure moreover produces plasmonic reverberation characteristics. This article designed and simulated a particular BPA to operate optical wavelength sensing applications, and Electromagnetic emission from the sun normally consider as the primary source of the optical wavelength. The most significant absorbance is 0.9999 $(99.99 \%)$ at $458.54 \mathrm{~nm}$ and ended up with $0.9552(95.52 \%)$ absorption at the $600 \mathrm{~nm}$ wavelength. The proposed design provides more than $0.8972(89.72 \%)$ absorbance in between the wavelength of $(450-600 \mathrm{~nm})$. To find the most effective absorption mechanisms, BPA changes the shape by regulating the unit cell parameters. Metal ground plane to minimize reflection and transmission to increase the specific absorption. To explain the maximum absorbance and BPA apparatuses change the shape of the unit cell by tuning parameters. A metal ground plane can reduce reflection and transmission to maximize absorption.

\section{Methodology of the Nano-Meta Absorber Design}

\section{A. Materials Choice}

The proposed designs consist of a star-shaped resonator in the top and a metal plane in the backside. The metal and dielectric layer is designed of tungsten and quartz (Fused), respectively. The most purposes behind picking the tungsten as a metal layer and the resonator are its high impedance coordinated to free space in the optical range and high natural losses that diminish the reflection and transmission near to zero. Lossless characteristic quartz (Fused) is chosen as the dielectric layer. Resonance characteristics support the design to impedance match, and tungsten exhibits unreasonable absorptivity inside the optical region. However, because almost lossless in the optical wavelength region, ignoring the absorption of whichever this one. This geometric structure plays a vital 
role in capturing the resonance wavelength. Therefore, change the design of the resonator using various forms of metamaterials, such as sawtooth and star-shaped cross hourglass-shaped also, an organic and inorganic film is used for adjusting absorption respectively (27), (28), (29). G. Ghosh gives the scattering condition, optical properties, and band structure of $\mathrm{W}$ and quartz (Fused), (30), (31).

\section{B. Geometric Layout of the Nano-Meta Unit Cell Structure}

The proposed design comprising with metal-dialectic-meta structure, and the hexagonal resonator is loaded with a star shape. The total length and width of the square shape structure is " $a=1000 \mathrm{~nm}$ ". Back layer (metal) thickness is " $\mathrm{tb}=150 \mathrm{~nm}$ ", dielectric layer " $\mathrm{td}=60 \mathrm{~nm}$ " and resonator " $\mathrm{tm}=10 \mathrm{~nm}$ ". The outer and inner radius of hexagonal shape " $R 1=200 \mathrm{~nm}$ " and " $R 2=150 \mathrm{~nm}$ ". The star shape radius is " $R \mathrm{~s}=100 \mathrm{~nm}$," The distance between the outer and inner edge of the hexagonal shape is denoted by "wh=25 nm". Back nurture metal layer thickness is higher than the skin depth, $(\delta)=(2 \rho / 2 \pi f \mu R \mu 0) 1 / 2$ to agreement near-zero transmission in the full optical region. This design is a rational symmetric structure, which will be proved below with sufficient data. However, the total thickness of the unit cell is $225 \mathrm{~nm}$, the ultrathin. These types of ultra-thin structures can be easily rolled on the thermophotovoltaic solar cell (STPV). The physical dimension of the proposed structure can be identified from Fig. 1, (a) three-dimensional view, (b) front-view, and (c) side-view from the x-axis, where dark red is considered as tungsten and light green color as quartz (Fused). From Table 1, the structure parameters can be easily understood. All the parameters and their value are demonstrated in Table 1.

Table 1. List of the parameter of this unit cell

\begin{tabular}{|c|c|c|c|c|c|c|c|c|}
\hline Parameters & $\mathrm{a}$ & $\mathrm{R} 1$ & $\mathrm{R} 2$ & $\mathrm{Rs}$ & $\mathrm{tb}$ & $\mathrm{td}$ & $\mathrm{tm}$ & wh \\
\hline Value $(\mathrm{nm})$ & 1000 & 200 & 150 & 100 & 150 & 60 & 10 & 25 \\
\hline
\end{tabular}

\section{Simulation Setup of Nano-Meta}

To continue the result and discussion, simulated a unit cell of the proposed design. Best electrical and magnetic (PEC, PMC) regarding plane $\mathrm{Y}-\mathrm{Z}$ and $\mathrm{X}-\mathrm{Z}$ within the reproduction cycle. This design simplest emphasizes that transverse electromagnetic waves (TEM) and z-axis are considered an open area in which frequencies will pass over the structure. The $\mathrm{x}$-axis and the $\mathrm{Y}$-axis are respectively taken as limitations to electric and magnetic limits. The boundary condition adequate to block close field inconvenience. Propagation direction followed electric and magnetic fields $\mathrm{X}, \mathrm{Y}$, and $\mathrm{Z}$ axes, severally. The electric fields, magnetic fields, and open spaces are opposite to one another. The structure is simulated by a study of microwave technology numerical computer simulation (CST) tools. The finite integration technique (FIT) is performed for data analysis in CST software, and the finite element method (FEM) of HFSS also helps validate simulation data. Moreover, data analysis through CST is validated in the results and discussion section. Fig. 1(a) shows the three-dimensional view of the submitted design, where (b) and (c) show the front view and side view, respectively. The proposed structure is square shape and length and width is defined as a where $\mathrm{a}=1000 \mathrm{~nm}$, the width of the dielectric layer is defined as td where $\mathrm{td}=60 \mathrm{~nm}$ respectively metal plane is defined as tb and its value, $t b=150 \mathrm{~nm}$. Dimensions with the parameter value are best achieved using genetic algorithm (GA) tools and parameter sweeps. 


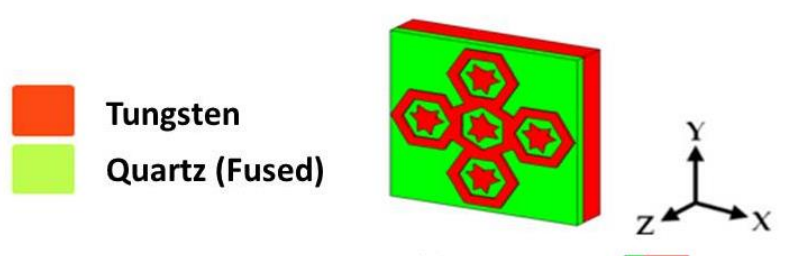

(a)

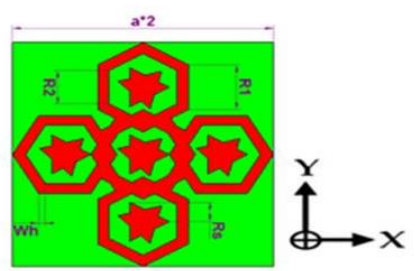

(b)

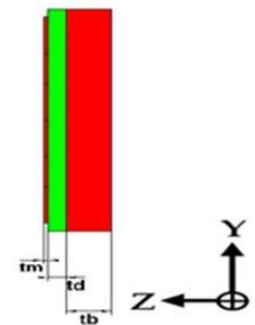

(c)

Figure 1(a) Three-dimensional view of the unit cell, (b) Front view, and (c) Side view

\section{Methodology for Calculation of Absorbance}

The actual permittivity and permeability take out from the S parameters by using Nicolson Ross Wire (NRW) (32) equation. From the $\mathrm{S}$ parameters, achieve reflection, transmission magnitudes and wave phase, respectively. Equation (1) helps to evaluate the response of the absorption of $A(\omega)$ the proposed structure.

$$
A(\omega)=1-R(\omega)-T(\omega)=1-\left|S_{11}^{2}(\omega)\right|^{2}-\left|S_{21}^{2}(\omega)\right|^{2}
$$

Here, Reflection, $R(\omega)=\left|S_{11}^{2}\right|$ and Transmission, $T(\omega)=\left|S_{21}^{2}\right|$

The unit cell characteristics impedance $Z(\omega)$ and calculated from Equation (2) and Equation (3).

$$
\begin{aligned}
& Z(\omega)=\left\{\mu_{r}(\omega) \cdot \mu_{0} /\left[\varepsilon_{r}(\omega) \cdot \varepsilon_{0}\right]\right\}^{1 / 2} \\
& Z_{0}=\left(\mu_{r} / \varepsilon_{r}\right)^{1 / 2}=376.73 \approx 377 \Omega
\end{aligned}
$$

$\mu_{0}=$ Vacuum Permeability, $\varepsilon_{0}=$ Vacuum Permittivity

$\mu_{r}=$ Relative Permeability, and $\varepsilon_{r}=$ Relative Permittivity

Following the terms of the intrinsic impedance of free space impedance $\left(Z(\omega)=Z_{0}\right)$ by changing the resonator's geometric structure and the approximate shape of a structure, the absorption will usually be near to unity, otherwise unity. In addition, the absorption is continuously conversely corresponding to the reflectance and transmittance. Due to their dispersive properties, the frequencies rely on these values. Seeing that tungsten obstructs one of the most transmittances $T(\omega)$ so the transmittance $T(\omega)$ is merely too tiny. The absorption is symmetrical to reflection $R(\omega)$, so the absorption can be extracted by calculating Equation (4).

$A(\omega)=1-R(\omega)=1-\left|S_{11}^{2}(\omega)\right|^{2}$

Here, $\left|S_{11}^{2}(\omega)\right|^{2}=0$ as $T(\omega) \square 0$

\section{Results and Discussions}

\section{A. Absorption Characteristics Investigations of the MMA}


The tungsten has an extraordinary exhibition to impedance match in free space, and tungsten is also utilized as a metal plane in this structure. Accordingly, there is no tungsten surface plasmon in optical wavelengths. As seen, the value of $\left(Z_{0}\right)$ is barely lower than the value of $Z(\omega)$ and show near-perfect absorption. This kind of absorber has to perform as an excellent potential super absorber. These incidents are controlled through the designed structure and propose exceptional absorbance for excessive bandwidth. The back-layer metal plane wave incidents affected anticipate low transmission, and additional layers maintain the front-wave resonator and the dielectric layer. For low thickness characteristic, quartz (Fused) supports to reduce the distance of the wavelength. Loss of intrinsic characteristics of the front layer and the resonance characteristics of dielectric layers also help understand the impedance matching with the free space optical region. This singularity is caused by infiltration of the corresponding wave absorber of high performance and efficiency. In any case, these all wonders will display this type of greater absorption if utilized the evenly organized hexagonal-shape resonator. To obtain a high absorption in the incident wave to be kept by the structure of MMA. The resonator appears the wave from the first stop as metal screens back layer wave once more consequently. The front layer comprises a metal resonator conjointly with a dielectric substrate (quartz), and the back layer is composed of a metal plane (tungsten). Fig. 2(b) illustrates the front-layer subsidized greater to the absolute absorption while the back layer supports a supplementary. This study observed that the front-layer absorption was reliable when back layer retention in the suggested structure. Fig. 2 (a) appears the absorption, reflectance, and transmittance to the specified dimensions by tungsten and quartz (Fused). The design has $0.8972(89.72 \%)$ average absorption concerning 450-600 nm wavelengths and almost perfect absorption of $0.9000(99 \%)$ at $458.54 \mathrm{~nm}$. This design demonstrated more than $0.90(90 \%)$ absorbance between wideband 400-600nm.

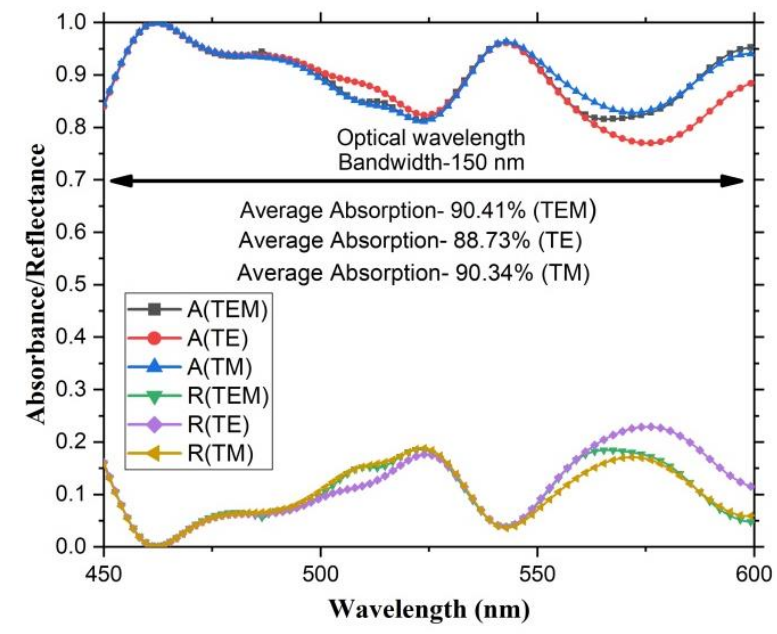

(a)

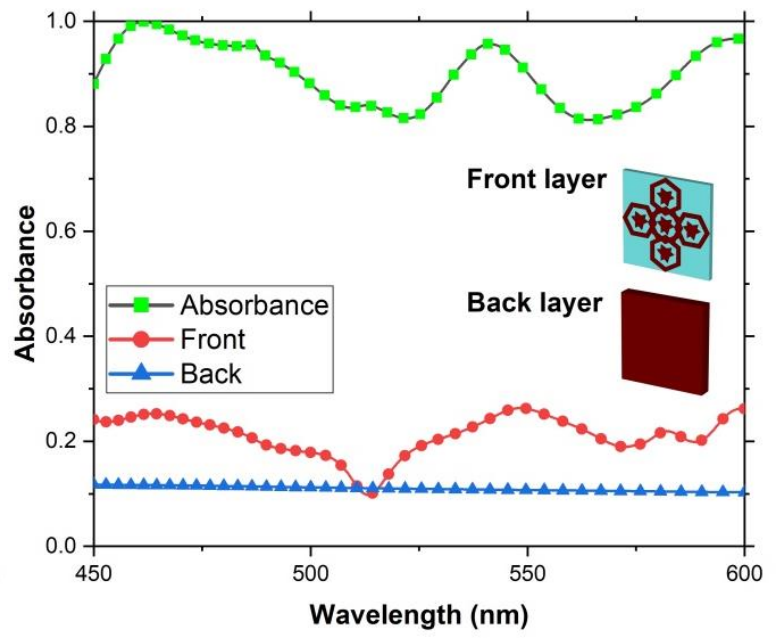

(b)

Figure 2(a) Graphical representations of absorbance, reflectance, and transmittance for TE, and TM, TEM mode. (b) Front and back-layer absorbance.

\section{B. Analysis co-polarization and cross-polarization with (PCR) of the MMA}

Absorption of the proposed absorber evaluated using equation (1) and (4). The unit cell should not function as a polarization converter and gives PCR value instead of absorbance. Demonstrated both co-polarization and crosspolarization component in Fig.3 (a) and calculated by using equation (5) and (6)

$$
\begin{aligned}
\left|S_{11}(\omega)\right|^{2} & =\left|S_{T E, T E}(\omega)\right|^{2}+\left|S_{T E, T M}(\omega)\right|^{2} \\
& =R_{y y}^{2}+R_{y x}^{2} \\
\left|S_{11}(\omega)\right|^{2} & =\left|S_{T M, T M}(\omega)\right|^{2}+\left|S_{T M, T E}(\omega)\right|^{2} \\
& =R_{x x}^{2}+R_{x y}^{2}
\end{aligned}
$$


PCR cell can be calculated by (7) or (8), which can be observed from Fig. 4 (b). PCR values of the proposed design are near zero for each $T E$ and $T M$ modes that care destroy the converter's polarization characteristics.

$$
\begin{aligned}
& P C R_{T E}=R_{y x}^{2} /\left(R_{y y}^{2}+R_{y x}^{2}\right) \\
& P C R_{T M}=R_{x y}^{2} /\left(R_{x x}^{2}+R_{x y}^{2}\right)
\end{aligned}
$$

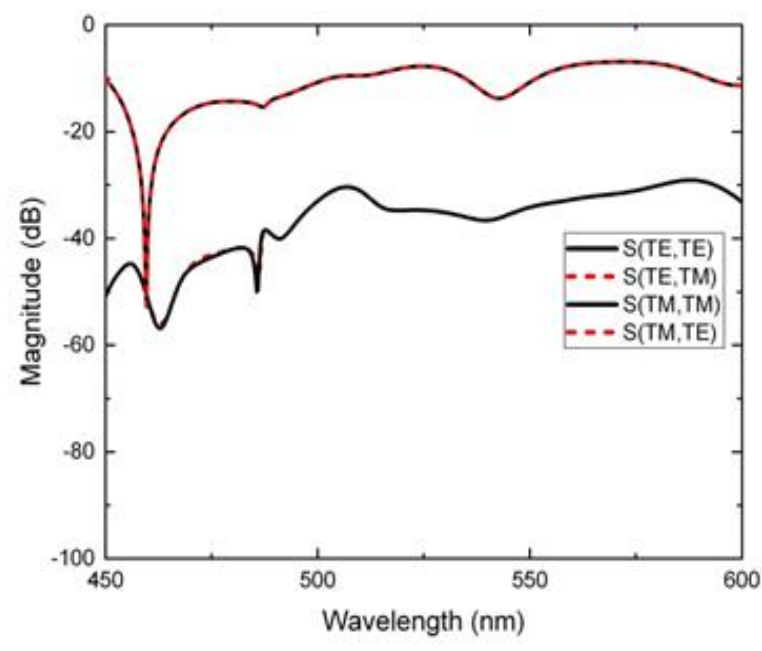

(a)

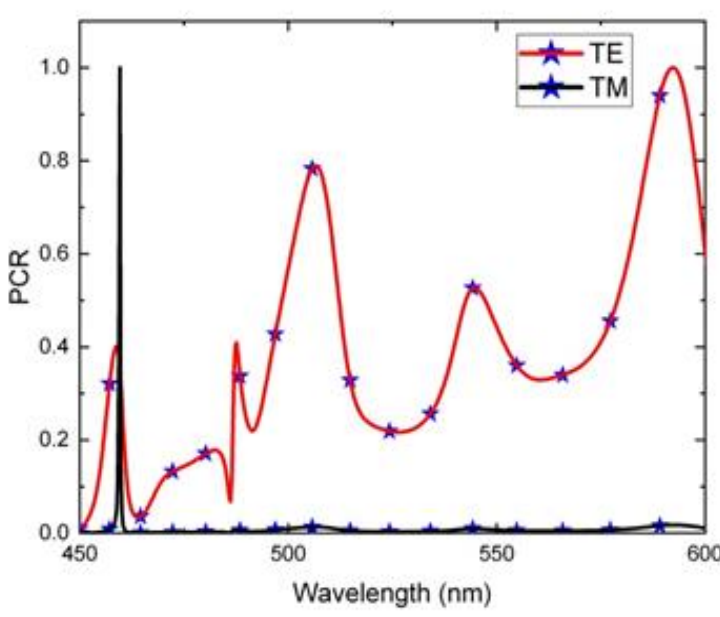

(b)

Figure 3. Co-polarization and cross-polarization magnitude (dB) for (a) Reflection coefficient ( $\left.\mathrm{S}_{11}\right)$ for $\mathrm{TE}$ and TM modes (b) PCR for both TE and TM mode of optical region

\section{Polarization and Incident Angle Stability of the Proposed MMA}

The proposed structure's absorption value with different polarization and angle of incidence to the TEM mode's polarization is defined. It shows that the structure is insensitive to polarization in between $0^{\circ}-90^{\circ}$ shows in Fig. 4 (a) and (b) respectively for transverse electric (TE) mode and transverse magnetic (TM) mode, additionally in Fig. 4(c) and (d) demonstrate the angular stability up to $45^{\circ}$. The symmetrical structure can console such independent behavior angles of MMA with high absorption with the suitable impedance matching. Besides, it can be used for optical sensors and does not need to change the sensor's position with the position of the wavelength of the source. The computational analysis found $0.8972(89.72 \%)$ absorption on average, better than $32 \%$ with lossy semiconductor/metal, potential as a solar energy harvester.
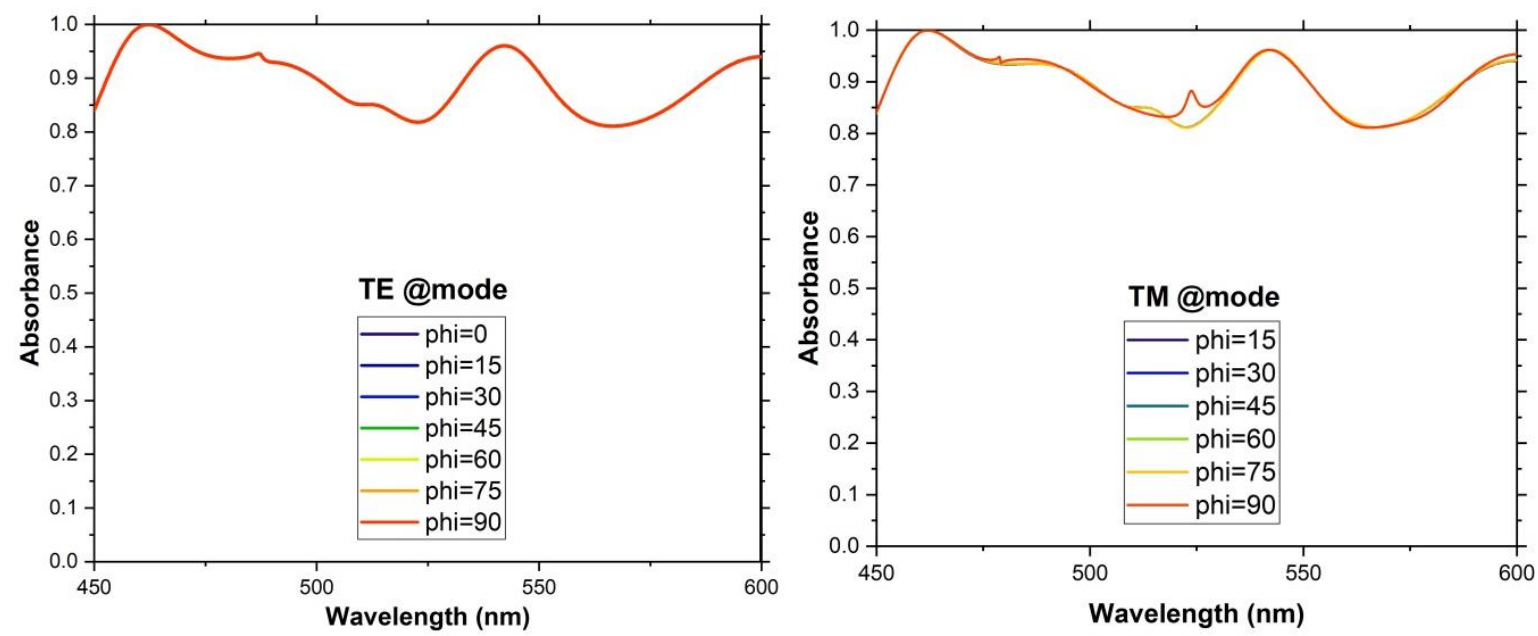
(a)

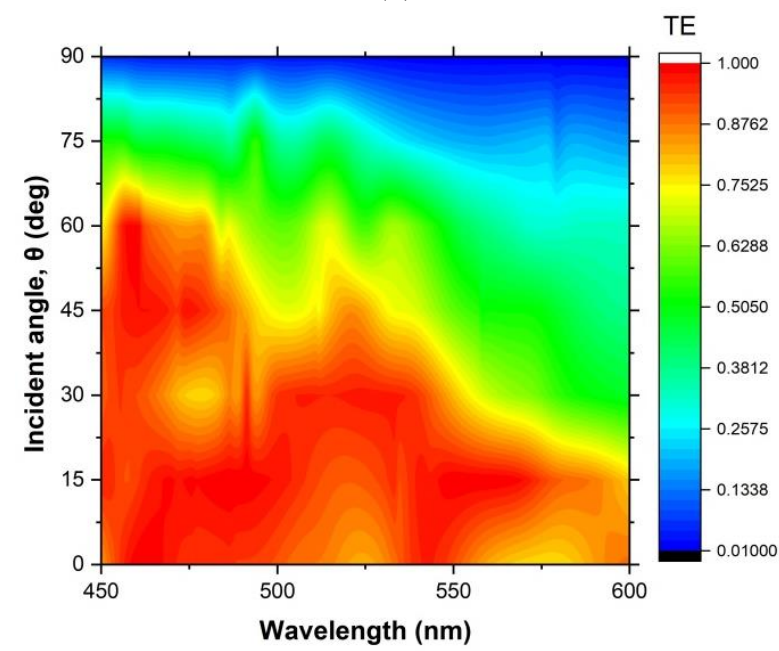

(c) (b)

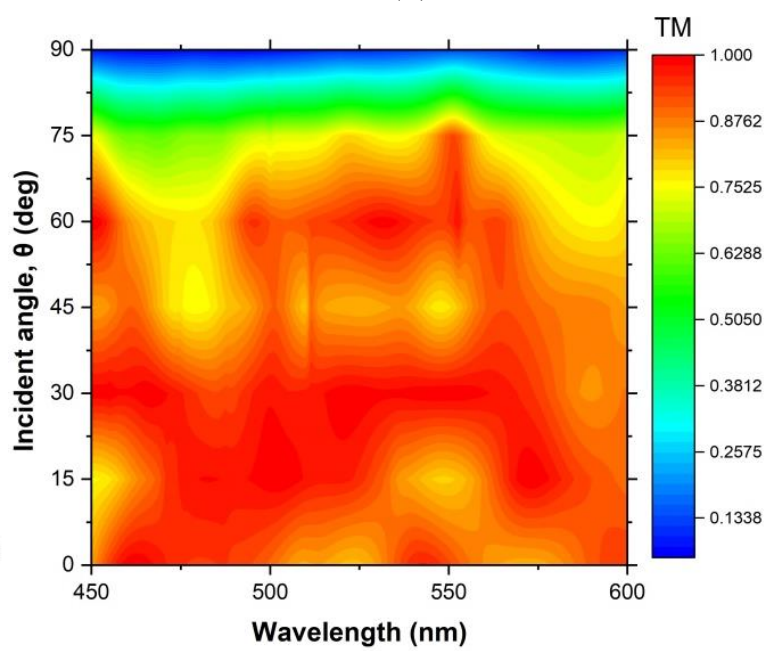

(d)

Figure 4. Absorption with distinctive polarization angle phi ( $\Phi$ (a) TE mode (b) TM mode and
Absorption with distinctive incident angle theta $(\Theta)$, (c) TE mode (d) TM mode.

\section{Geometric Parameter Sweep of the MMA Unit Cell}

Parameters of Table 1, sweep with their shape alter next to the table. The sweep parameter helps determine the absorbance of the most excellent design and utilization. The parameter sweep "R1", "R2", "Rs", "tm" and "td" were not disclosed here because they do not show significant results. Swapping the major parameters "R1" the external radius of the proposed hexagonal shape increases and decreased. The impact of changes $\mathrm{R} 1$ dimensional hexagonal 170-200 nm at $10 \mathrm{~nm}$ interim and shown in Fig. 5(a) specific changes include five steps and the adjusted difference between the hexagonal outer radius $10 \mathrm{~nm}-40 \mathrm{~nm}$. There are six hexagonal shapes presented in this design. The extent of absorption of all values of the deviation from the hexagonal radius is greater than $90 \%$ identical, and at the resonance frequency, it gets $0.999(99.99 \%)$ absorbance. The perfect absorbance finds at a radius of $40 \mathrm{~nm}$, which seemed to be at $\mathrm{R} 1=200 \mathrm{~nm}$. Particular resonance wavelength shift with the enhancements made to the outer radius of the hexagonal shape. The second parameter is changed to "R2" by changing these parameters, the hexagonal shape's inner radius increased and decreases. There was a minor up-down in the initial and end value whenever it changed the particular parameter "R2" in Fig. 5(b). The impacts of the hexagonal inner dimension R2 vary from 140-170 nm at intervals of $10 \mathrm{~nm}$. This specific alter included five steps and changes the contrast between the hexagonal internal sweep $10 \mathrm{~nm}-40 \mathrm{~nm}$. The resonance frequency varies insufficient but continuously stays up to $0.90(90 \%)$. The absorption at the resonance frequency is $0.9999(99.99 \%)$, and the magnitude of the absorption at operating wavelength $450-600 \mathrm{~nm}$ more than 0.90 (90\%). The perfect absorbance finds at the radius of $20 \mathrm{~nm}$, which seemed to be at R2 $=150 \mathrm{~nm}$. Continuous increments of "R2" decreased the self-inductance but increased the typical inductance and the metal layer since the resonator shape expanded. These types of shared inductance developed the reverberation wavelength directly. These parametric types consider making a difference to all of us to decide the resonator's ideal measure. The third parameter, "Rs" by varying these parameters, increased and decreased the star shape radius. The initial value slightly changes with Rs value. Still, the end value is considerable up-down, which shows in Fig. 5(c). The impacts of star measurement Rs alter from 90-120 $\mathrm{nm}$ at an interval of $10 \mathrm{~nm}$ and the finest esteem found was at $\mathrm{Rs}=100 \mathrm{~nm}$ and the resonance frequency varies insufficiently but remains permanently up to $0.90(90 \%)$. 


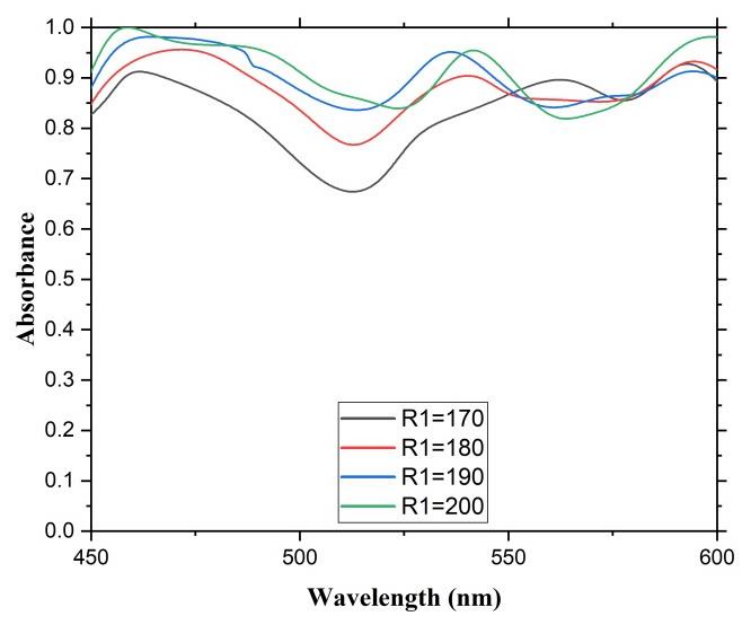

(a)

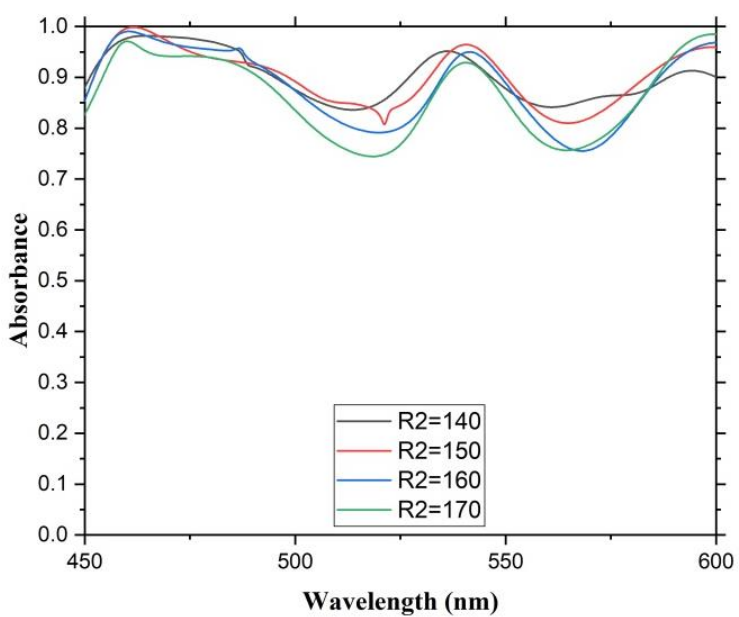

(b)

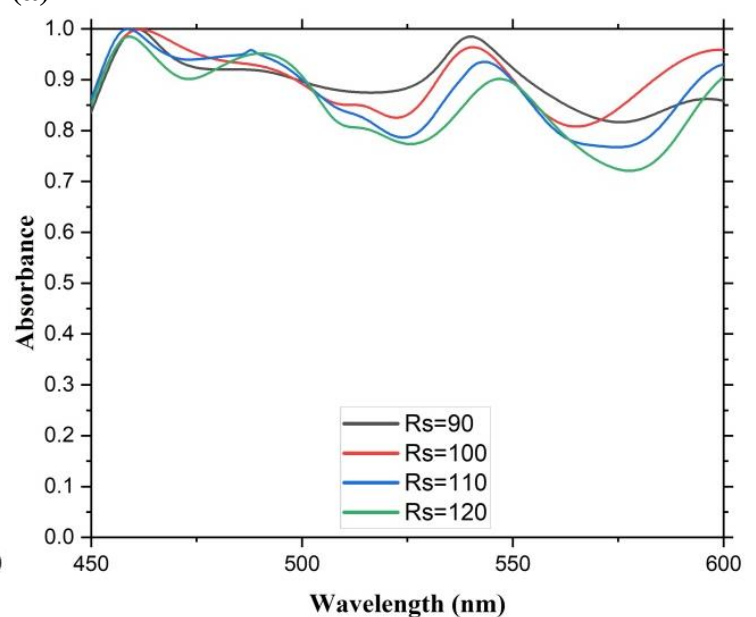

(c)

Figure 5. Wavelength response to a parameter sweep (a) Outer radius of hexagonal shape "R1", (b) Inner radius of hexagonal shape "R2" and (c) Radius of star shape "Rs"

A unique platform was apparent within the variety associated with the parameter "td", which usually denotes the thickness related to the dielectric substance displayed in Fig. 6(a). In this article, quartz (Fused) is used as dielectric materials. Modifying this value from 30-60 nm, observed a most important adjustment in the absorbance and even the resonance wavelength more sweep. Thicknesses of dielectric level 30, 40, 50 and 60 appeared with reverberation values of $0.9584(95.84 \%), 0.9220(92.20 \%), 0.9875(98.75 \%)$ in addition to $0.9961(99.61 \%)$, individually. The dielectric layer width modified the reverberation frequency straightly at 461.46, 476.44, 600, and $459.77 \mathrm{~nm}$. This specific dielectric layer thickness fluctuation may well be clarified by the resonator capacitance alter with the variable alter. The capacitance substance in between the metallic and resonator was alternatively by the particular dielectric layer width. Typically, the structure's identical capacitance has been decreased as the higher metal structure capacitance is in sequence with others. Diminish within capacitance expanded the resonance frequency. This singularity may be utilized inside optical sensors or identify the thickness of any kind of dielectric layer. Almost all efficient results find out at td equal $60 \mathrm{~nm}$. The final and imperative parameter sweep included sweeping the resonator's thickness and "tm" which has been modified from 5-20 nm with an interim associated with $5 \mathrm{~nm}$, as made an appearance in Fig. 6(b) then the width of the resonator increased the particular resonance wavelength. In this specific study, each strip illuminated to an electric field component of the electromagnetic field had an inductance. Moreover, the resonator and metallic made of capacitance and inductance are relatively in thickness, and all the more important echo frequency of $0.90(90 \%)$ at almost all thicknesses. 


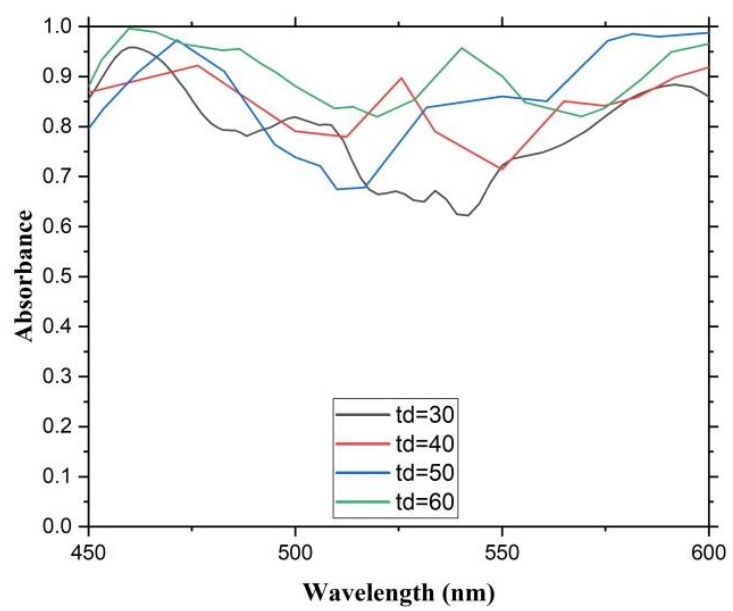

(a)

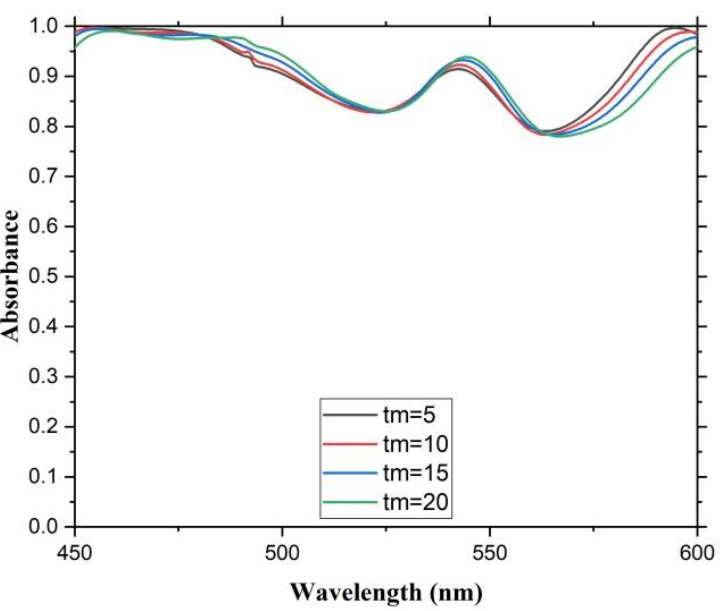

(b)

Figure 6. (a) Dielectric layer thickness "td" and (b) Resonator layer thickness "tm"

\section{E. Absorption with Distinctive Dielectric Materials of the MMA}

Dielectric materials change with five different dielectric materials and then simulation, we get the most acceptable result with quartz (Fused) appeared in Fig.7. Silicon nitride $\left(\mathrm{Si}_{3} \mathrm{~N}_{4}\right)$ produced an appearance associated with direct absorbance. This property permits this kind of structure to get unconstrained since a visible light detecting for absorbance numerous appears for each coloring of sunshine. It can be utilized as a great undetectable cloak for a proficiently light spectrum of its absorbance. Two results with gallium arsenide and amorphous silicon showed the half-power absorbance typically through a huge number of wavelengths, which could generally be utilized to fifty percent power of the application solar cells. The grapheme offers over $0.9124(91.24 \%)$ absorbance throughout the all-region. The modification of materials appears additionally to change the application related to the design of similar metamaterials.

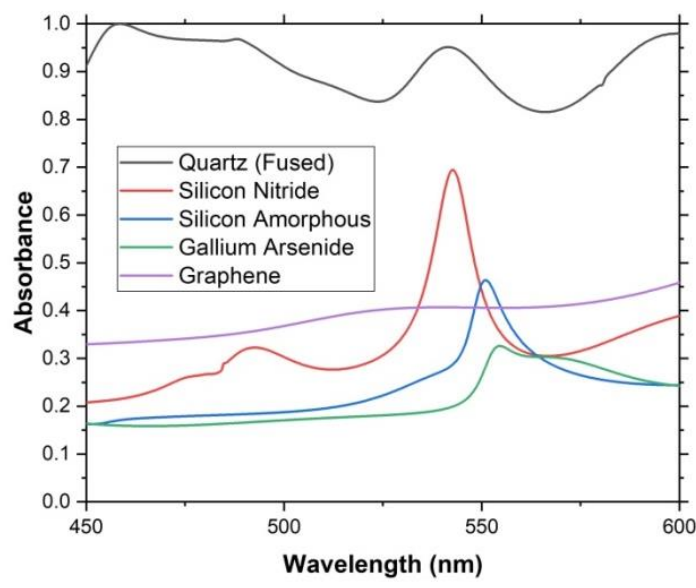

Figure 7. Different materials absorption analysis

\section{F. Metamaterial characterization of the proposed absorber}


Metamaterial characterization method refer to NRW (32) is related to $S_{11}$ and $S_{21}$ expressed as complex relation in equation (9) where $\mathrm{s}_{0}= \pm 1$ and $M=\sqrt{\frac{1-\left|S_{11}\right|^{2}}{\left|S_{11}\right|^{2}}}$. The polarity of $\mathrm{s}_{0}$ changes, whereas $S_{11}$ remains unchanged. So, $S_{21}=j s_{0} S_{11} M$

polarization angle $\varphi$ can change from $+\pi$ to $-\pi$ because of four (4) distinct polarity variations. In free space, both perpendicular and parallel polarization in basic waveguide mode (for example, $\mathrm{TE}_{10}$ ) shows a relationship between the square of wave vector $|k|^{2}$ and susceptibility tensor $\kappa$ of oblique incident wave (33). The reported article reveals that $|k|^{2}<\kappa^{2}$ a real part of $\varepsilon_{\mathrm{r}}$ and $\mu_{\mathrm{r}}$ should have the same sign, where the imaginary part will have an opposite sign for perpendicular polarization. Similarly, the same scenario applicable for parallel polarization, if $|k|^{2}<\sin ^{2} \theta_{0}$ where $\theta_{0}$ is the incident angle. Herein, $\varepsilon_{\mathrm{r}}$ in Fig. 12a shows real and imaginary parts almost different, except for lower wavelengths such as $468 \mathrm{~nm}$. The quartz substrate's tensor value is typically high compared to metal like tungsten and dipole tensor point deflects the visible spectrum's in higher wavelength. Thus, the proposed absorber's significant wavelength illustrates the opposite values of dielectric parameters (Fig

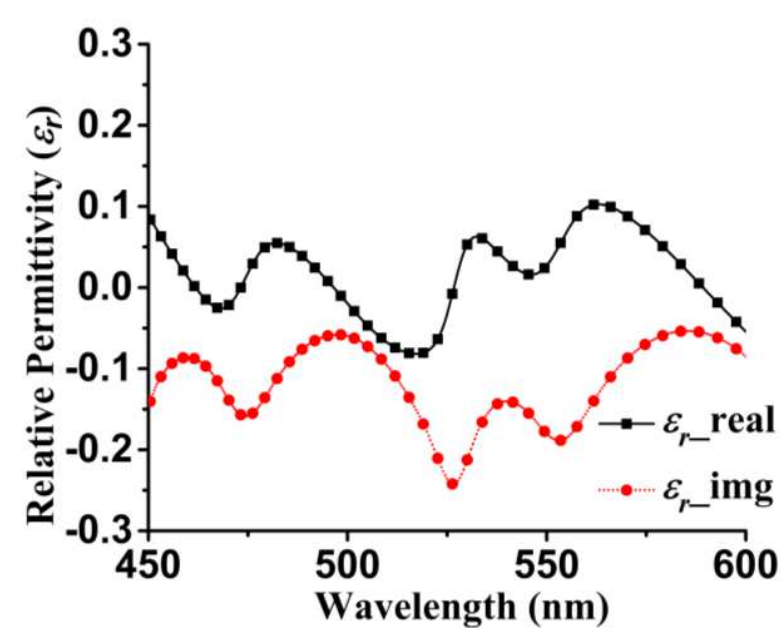

(a)

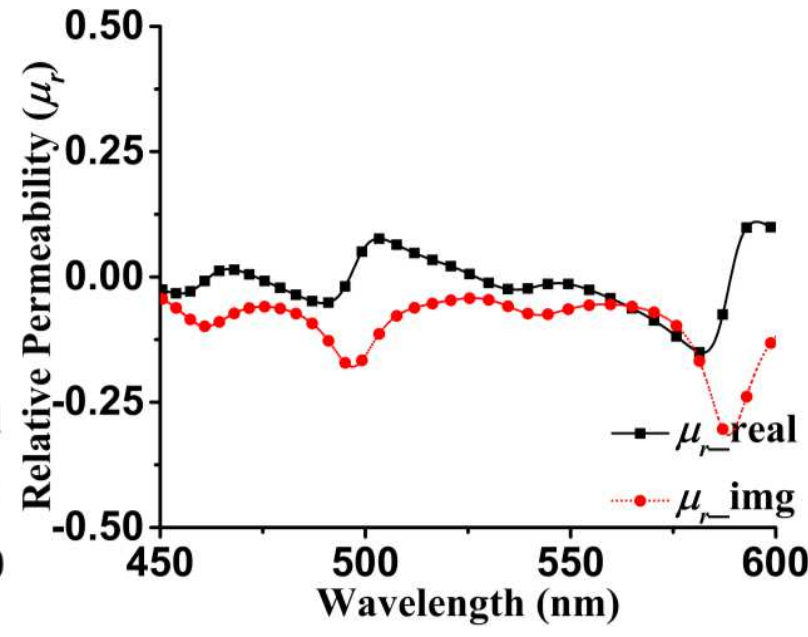

(b)

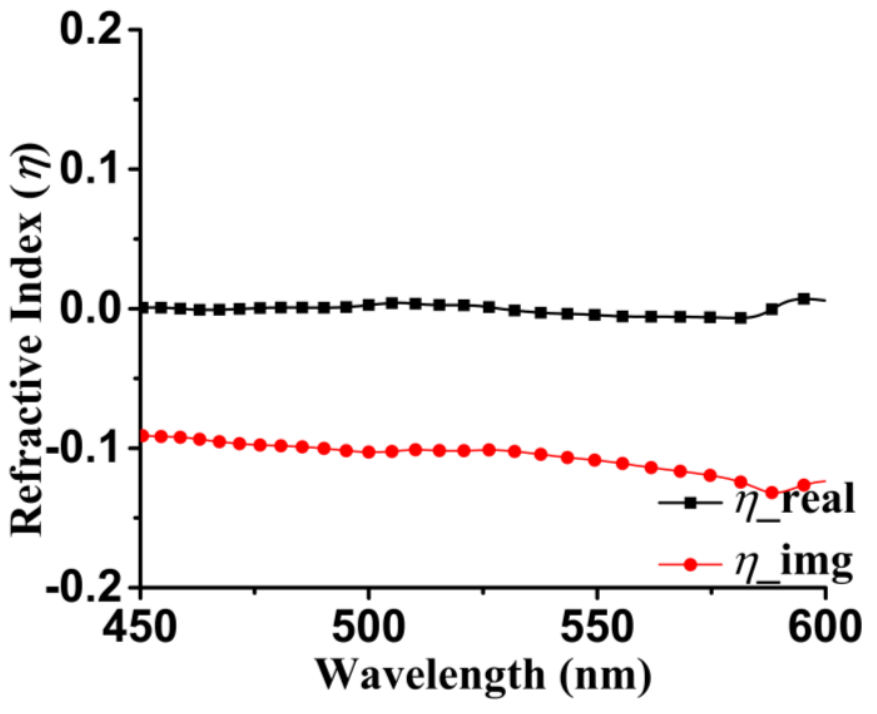


Figure 8. Proposed metamaterial absorber dielectric properties characterization (a) Relative Permittivity (b) Relative Permeability (c) Refractive Index

$8 \mathrm{a}$ and $\mathrm{b}$ ). However, a significant negative $\varepsilon_{\mathrm{r}}$ of -0.08 exists between 500-526 nm wavelength, whereas negative $\mu_{\mathrm{r}}$ achieved at $581 \mathrm{~nm}$ with a value of -0.15 . Eventually, refractive index $(\eta)$, all over the operational spectrum remains approximately close to zero (between $460 \mathrm{~nm}$ to $600 \mathrm{~nm}$ ) expect between $450-458 \mathrm{~nm}$ due to the relationship of $\eta$ and dielectric properties parameters (Fig. 8c). Therefore, based on computational analysis, the proposed absorber can be characterized as Zero Indexed Metamaterial (ZIM).

\section{G. Electric Field, Magnetic Field and Surface Current Analysis}

The electromagnetic field and surface current distribution from $458.54 \mathrm{~nm}, 480 \mathrm{~nm}$ and $540 \mathrm{~nm}$ wavelength are presented in Fig. 13 to understand the proposed design's propagation energy. The electric field, magnetic field, and surface current distribution are shown in Fig. 13(a), (b) and (c) for three wavelengths presented, respectively. Complete dissipation shown on the E-field resonance wavelength $(458.54 \mathrm{~nm})$ has the most significant absorbance with high resonator excitation. The magnetic field had an anti-parallel conveyance concerning the coupling impact of the resonator and metal. It appeared with exceptional esteem at $458.54 \mathrm{~nm}$ but was generally energized by the wavelength at each point. Fig. 13 seems the surface current conveyance, $480 \mathrm{~nm}$, had a less strong surface current taken after by $458.54 \mathrm{~nm}$, separately. Fig. 13(c) illustrated that surface current dispersion was exceptionally significant at $458.54 \mathrm{~nm}$. The back-layer was, for the most part, less energized than the front-layer in all three phenomena.

$458.54 \mathrm{~nm}$

$480 \mathrm{~nm}$

$540 \mathrm{~nm}$

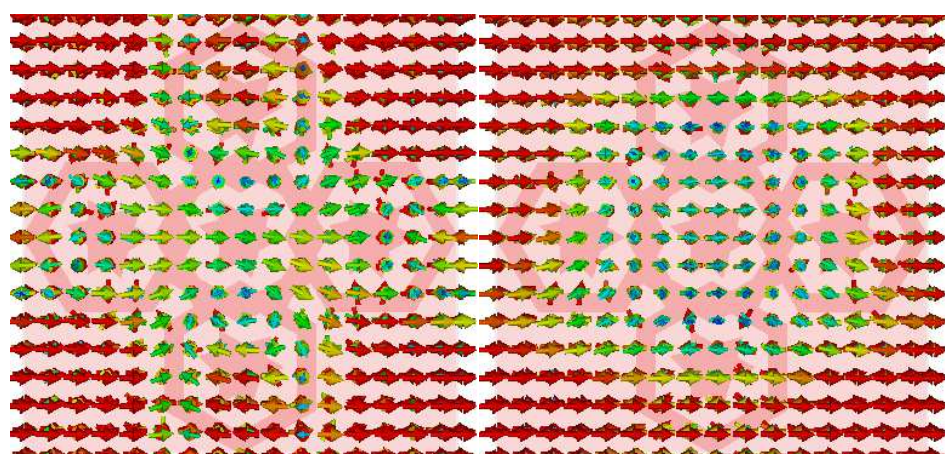

(a)

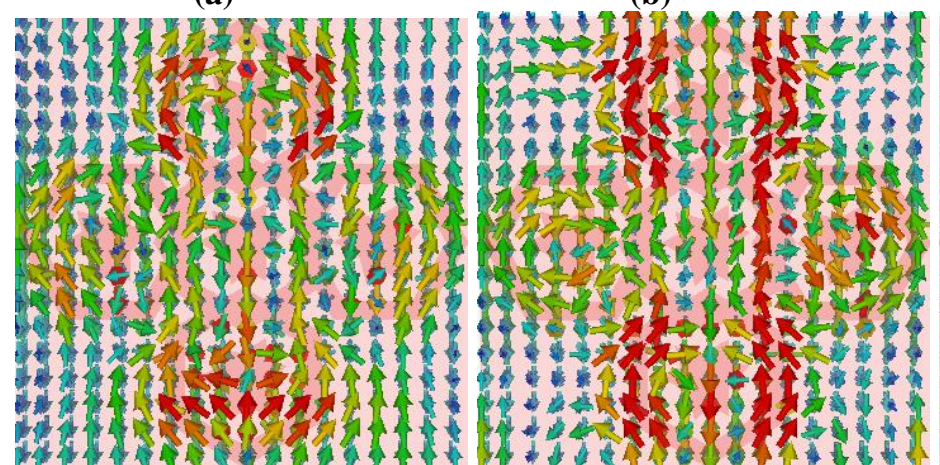

(d) (e)

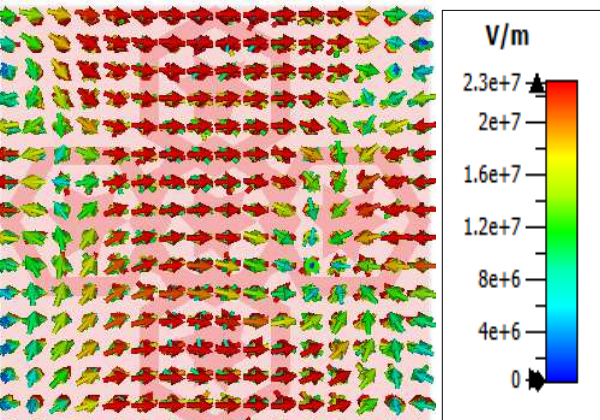

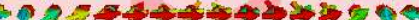

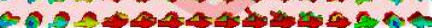
(c)

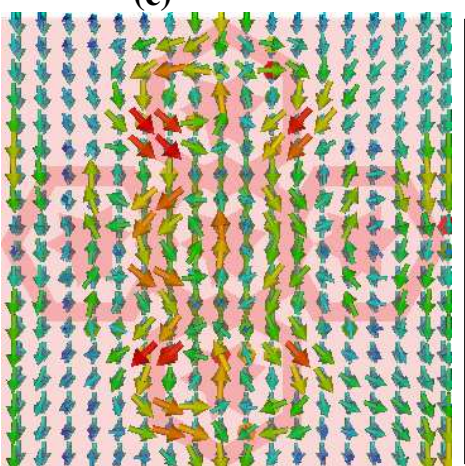

$\mathrm{A} / \mathrm{m}$

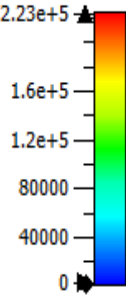




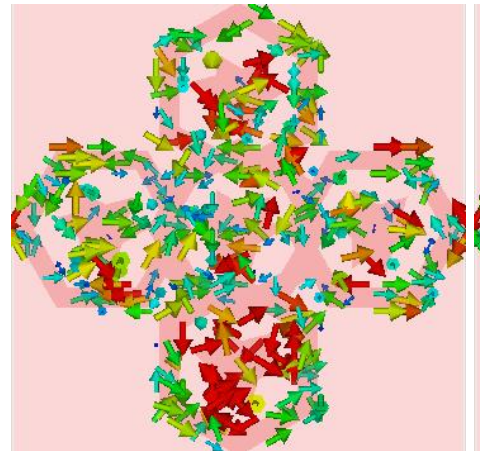

(g)

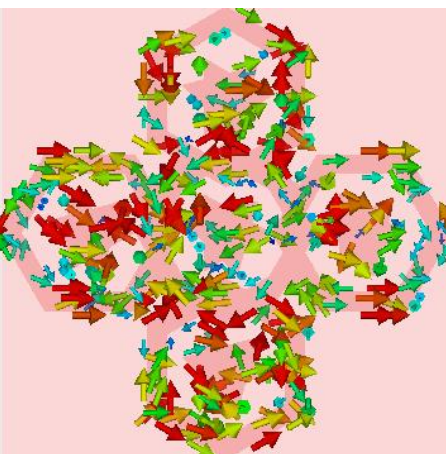

(h)

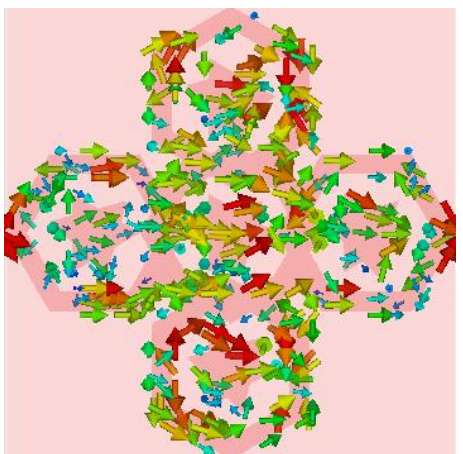

(i)

Figure 9. Surface current distribution of proposed MMA: a, b, c ; d, e, f;g, h, I, respectively demonstrate $(E),(H)$ and surface current distribution of the resonator at $458.54 \mathrm{~nm}, 480 \mathrm{~nm}, 540 \mathrm{~nm}$.

\section{H. Demonstrates the Simulated Data with CST and HFSS for Validation}

To validate the proposed structure, demonstrate the simulation data with different simulation software. Fig. 10(a) and (b) demonstrates the comparison between the CST and HFSS data with $\mathrm{S}_{11}$ parameter and absorption. The maximum absorption stays nearly equal at 0.9999 (99.99\%), and the average absorption is above $0.8972(89.72 \%)$ in the visible wavelength.

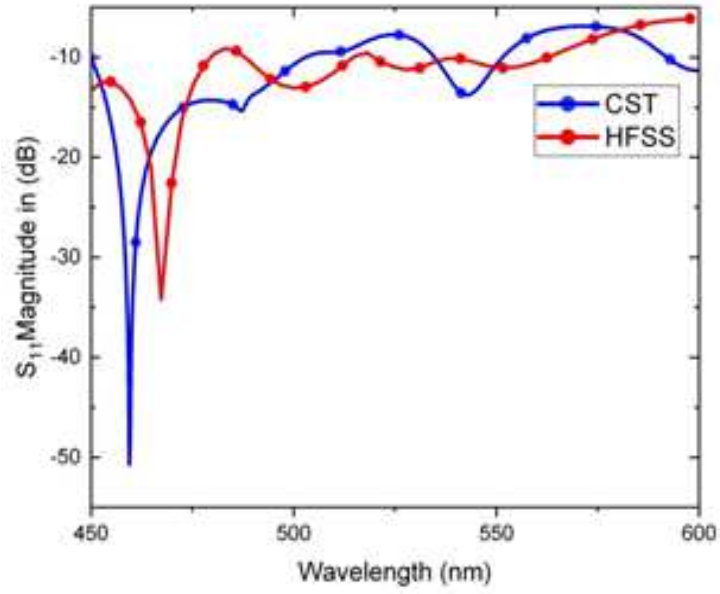

(a)

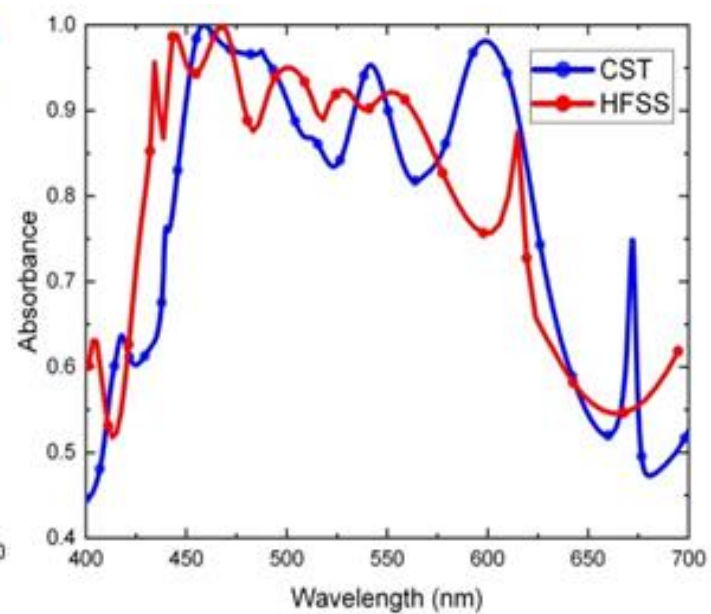

(b)

Figure 10. Comparison of different simulation methods with the S11 parameter (a) Magnitude in dB (b) Comparison of absorption with different simulation methods

Additionally, this study observes that the magnitude of the typically $S_{11}$ parameter likewise synchronized quite well, which is an objective behind the match inside absorbance. The back-layer has been made with tungsten, and the impedance coordinate with the free space, particularly the optical frequency, was the primary explanation behind this specific. Capacitive and inductive properties in front-layer from the resonator and lossless characteristics quartz (Fused) were the cause for the absorption. Although overlooked silicon dioxide assimilation, that shows superb absorption consisting of a metal resonator. These design Comparisons with tungsten and quartz (Fused) also simulated data verified by the finite element method (FEM) in ANSYS with high-frequency software (HFSS).

\section{Comparative study}

The proposed design is finding a remarkable absorption rate compared with the previous work. The evaluation of the proposed design and the previous work is demonstrated in Table2. Culminate optical wavelength absorbers that could function in full areas are once in a great exceedingly large area. A rare absorbance, almost perfect than 
$0.8972(89.72 \%)$ at all points with $0.9999(99.99 \%)$ peak points throughout optical wavelengths, is identified in the proposed design. A fantastic impedance organize of tungsten is perhaps the most reason for incredible absorbance. This design utilizes a distinctive geometric structure compact with five hexagonal shapes, improving the property of absorption. The resonator and the back-layer metal are high with the equivalent capacitance. In terms involving the unique softening level of tungsten as well as quartz (Fused), effectiveness, and operational area contrasted and different materials utilized in Table 2, makes this structure a sole.

Table 2. Comparison of the proposed research with existing research

\begin{tabular}{|c|c|c|c|c|c|c|}
\hline $\begin{array}{l}\text { Cited } \\
\text { paper }\end{array}$ & $\begin{array}{c}\text { Layers } \\
\text { Numbers }\end{array}$ & Material used & Substrate & $\begin{array}{l}\text { Bandwidth } \\
\text { (nm) }\end{array}$ & $\begin{array}{c}\text { Absorption } \\
(\%)\end{array}$ & $\begin{array}{c}\text { Peak } \\
\text { value } \\
(\%)\end{array}$ \\
\hline (7) & Three & Silver & $\begin{array}{l}\text { Fused } \\
\text { Quartz }\end{array}$ & 300 & Above 90 & 98 \\
\hline (16) & Four & $\begin{array}{c}\mathrm{Cu} / \mathrm{Si} 3 \mathrm{~N} 4 / \mathrm{Cu} \\
\text { stack }\end{array}$ & Silicon & 300 & Above 80 & 97 \\
\hline (21) & Three & Silver & $\begin{array}{l}\text { Silicon } \\
\text { Dioxide }\end{array}$ & 300 & Above 71 & 95 \\
\hline (24) & $\begin{array}{c}\text { Periodic } \\
\text { array }\end{array}$ & Gold/Silicon & Lattice & 310 & Above 80 & 99 \\
\hline (26) & Three & Silver & $\begin{array}{l}\text { Silicon } \\
\text { Dioxide }\end{array}$ & 300 & Above 80 & 98 \\
\hline (33) & Three & Tungsten & $\begin{array}{l}\text { Silicon } \\
\text { Dioxide }\end{array}$ & 308 & Above 91.24 & 99.99 \\
\hline Proposed & Three & Tungsten & $\begin{array}{l}\text { Quartz } \\
\text { (Fused) }\end{array}$ & 177.91 & Above 80 & 99.99 \\
\hline
\end{tabular}

\section{Conclusion}

Zero Index MMA incredibly broadband for 450-600 nm of visible wavelengths has been designed inside this specific research. The proposed ZIM absorber structures demonstrate a very extensive wide bandwidth and demonstrated almost perfect absorbance with $99.99 \%$. Furthermore, the proposed nanostructure is presented with polarization-independent behavior and angle of incidence with an average of $0.8972(89.72 \%)$ absorption. Due to the adaption of tungsten and lossless characteristics quartz (Fused), perfect absorption is taken out with proposed structure. This research is conducted along with four other dielectric material and find variations of valuable results. Absorbance is linear with a wavelength shift if $\mathrm{Si}_{3} \mathrm{~N}_{4}$ and glass (Pyrex) are used since dielectric substrates. This ZIM absorber can be used as an optical sensor for a shift in the resonance and changes in the dielectric layer and the resonator's thickness. A high combustion point would help withstand very high temperatures naturally absorb photon's design of better energy sources. The points outstanding combustion of materials applied to this study adds to the substantial absorbance usually associated with broadband absorber, which may also be effectively used in solar cells, optical sensors, a level unseen real, color image, and thermal imaging applications.

\section{Funding:}

Conflicts of Interest: The authors declare no conflict of interest.

Authors contribution: Authors contribution: Ismail Hossain, Ahasanul Hoque and Md. Samsuzzaman contributed significantly to the planning, analysis of the findings, preparation, and paper writing. Ismail Hossain, Ali F. Almutairi, Md. Samsuzzaman and Mohammad Tariqul Islam carried out the result analysis then review of the desired results. Ali F. Almutairi and Mohammad Tariqul Islam oversaw the entire investigation and financing. Md. Samsuzzaman, Ali F. Almutairi and Mandeep Singh have reviewed the text, concept optimization, 
characterization, empirical findings, and critical recommendations. Ali F. Almutairi, Mohammad Tariqul Islam and Mandeep Singh have updated the article judgmentally and reviewed useful recommendations for relevant intellectual material. All authors have read the manuscript and agreed to publish.

\section{Reference}

1. Veselago VG. The Electrodynamics of Substances with Simultaneously Negative Values of Img Align= Absmiddle Alt= $\epsilon$ Eps/Img and $\mu$. Physics-Uspekhi. 1968;10(4):509-14.

2. Smith DR, Padilla WJ, Vier D, Nemat-Nasser SC, Schultz S. Composite medium with simultaneously negative permeability and permittivity. Physical review letters. 2000;84(18):4184.

3. Smith D, Vier D, Koschny T, Soukoulis C. Electromagnetic parameter retrieval from inhomogeneous metamaterials. Physical review E. 2005;71(3):036617.

4. Hasan MM, Faruque MRI, Islam MT. Dual band metamaterial antenna for LTE/bluetooth/WiMAX system. Scientific reports. 2018;8(1):1-17.

5. Fan F, Zhang X, Li S, Deng D, Wang N, Zhang H, et al. Terahertz transmission and sensing properties of microstructured PMMA tube waveguide. Optics express. 2015;23(21):27204-12.

6. Islam SS, Faruque MRI, Islam MT. A near zero refractive index metamaterial for electromagnetic invisibility cloaking operation. Materials. 2015;8(8):4790-804.

7. Zhang X, Fan Y, Qi L, Li H. Broadband plasmonic metamaterial absorber with fish-scale structure at visible frequencies. Optical Materials Express. 2016;6(7):2448-57.

8. Jadeja R, Charola S, Patel SK, Parmar J, Ladumor M, Nguyen TK, et al. Numerical investigation of graphene-based efficient and broadband metasurface for terahertz solar absorber. Journal of Materials Science. 2020;55(8):3462-9.

9. Duan G, Schalch J, Zhao X, Zhang J, Averitt R, Zhang X, editors. An air-spacer terahertz metamaterial perfect absorber for sensing and detection applications. 2017 19th International Conference on Solid-State Sensors, Actuators and Microsystems (TRANSDUCERS); 2017: IEEE.

10. Watts CM, Liu X, Padilla WJ. Metamaterial electromagnetic wave absorbers (adv. mater. 23/2012). Advanced Materials. 2012;24(23):OP181-OP.

11. Lee Y, Tuong P, Zheng H, Rhee J, Jang W. An application of metamaterials: perfect absorbers. Journal of the Korean Physical Society. 2012;60(8):1203-6.

12. Landy NI, Sajuyigbe S, Mock JJ, Smith DR, Padilla WJ. Perfect metamaterial absorber. Physical review letters. 2008;100(20):207402.

13. Tao H, Landy NI, Bingham CM, Zhang X, Averitt RD, Padilla WJ. A metamaterial absorber for the terahertz regime: design, fabrication and characterization. Optics express. 2008;16(10):7181-8.

14. Nguyen TT, Lim S. Wide incidence angle-insensitive metamaterial absorber for both TE and TM polarization using eight-circular-sector. Scientific Reports. 2017;7(1):1-11.

15. Zhang Y, Li T, Chen Q, Zhang H, O'Hara JF, Abele E, et al. Independently tunable dual-band perfect absorber based on graphene at mid-infrared frequencies. Scientific reports. 2015;5:18463.

16. Zhu P, Jay Guo L. High performance broadband absorber in the visible band by engineered dispersion and geometry of a metal-dielectric-metal stack. Applied Physics Letters. 2012;101(24):241116.

17. Duan X, Chen S, Liu W, Cheng H, Li Z, Tian J. Polarization-insensitive and wide-angle broadband nearly perfect absorber by tunable planar metamaterials in the visible regime. Journal of Optics. 2014;16(12):125107.

18. Rufangura P, Sabah C. Dual-band perfect metamaterial absorber for solar cell applications. Vacuum. 2015;120:68-74.

19. Azad AK, Kort-Kamp WJ, Sykora M, Weisse-Bernstein NR, Luk TS, Taylor AJ, et al. Metasurface broadband solar absorber. Scientific reports. 2016;6:20347.

20. Heidari M, Sedighy S. Broadband wide-angle polarization-insensitive metasurface solar absorber. JOSA A. 2018;35(4):522-5.

21. Aydin K, Ferry VE, Briggs RM, Atwater HA. Broadband polarization-independent resonant light absorption using ultrathin plasmonic super absorbers. Nature communications. 2011;2(1):1-7.

22. Li C, Fan H, Dai Q, Wei Z, Lan S, Liu H. Multipole resonance in arrays of diamond dielectric: a metamaterial perfect absorber in the visible regime. Nanomaterials. 2019;9(9):1222.

23. Khan AD, Khan AD, Khan SD, Noman M. Light absorption enhancement in tri-layered composite metasurface absorber for solar cell applications. Optical Materials. 2018;84:195-8.

24. Hoa NTQ, Tung PD, Lam PH, Dung ND, Quang NH. Numerical study of an ultrabroadband, wide-angle, polarization-insensitivity metamaterial absorber in the visible region. Journal of Electronic Materials. 2018;47(5):2634-9.

25. Abdulkarim YI, Deng L, Muhammad FF, He L. Enhanced light absorption in the organic thin films by coating cross-shaped metamaterial resonators onto the active layers. Results in Physics. 2019;13:102338. 
26. Butun S, Aydin K. Structurally tunable resonant absorption bands in ultrathin broadband plasmonic absorbers. Optics express. 2014;22(16):19457-68.

27. Qi Y, Zhang Y, Liu C, Zhang T, Zhang B, Wang L, et al. A Tunable Terahertz Metamaterial Absorber Composed of Hourglass-Shaped Graphene Arrays. Nanomaterials. 2020;10(3):533.

28. Cheng YZ, Cheng ZZ, Mao XS, Gong RZ. Ultra-thin multi-band polarization-insensitive microwave metamaterial absorber based on multiple-order responses using a single resonator structure. Materials. 2017;10(11):1241.

29. Huang H-L, Xia H, Guo Z-B, Xie D, Li H-J. Design of broadband metamaterial absorbers for permittivity sensitivity and solar cell application. Chinese Physics Letters. 2017;34(11):117801.

30. Ghosh G. Dispersion-equation coefficients for the refractive index and birefringence of calcite and quartz crystals. Optics communications. 1999;163(1-3):95-102.

31. Schneider PM, Fowler WB. Band structure and optical properties of silicon dioxide. Physical Review Letters. 1976;36(8):425.

32. Rothwell EJ, Frasch JL, Ellison SM, Chahal P, Ouedraogo RO. Analysis of the Nicolson-Ross-Weir method for characterizing the electromagnetic properties of engineered materials. Progress In Electromagnetics Research. 2016;157:31-47.

33. Mahmud S, Islam SS, Mat K, Chowdhury ME, Rmili H, Islam MT. Design and parametric analysis of a wide-angle polarization-insensitive metamaterial absorber with a star shape resonator for optical wavelength applications. Results in Physics. 2020;18:103259. 
Figures

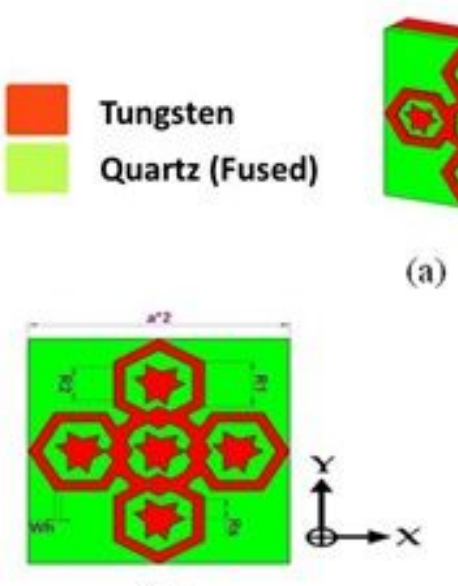

(b)
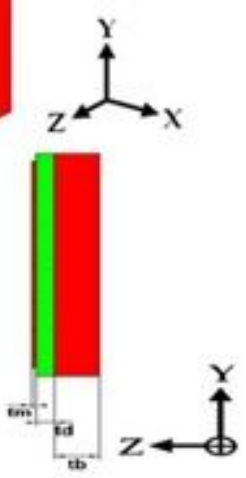

(c)

\section{Figure 1}

(a) Three-dimensional view of the unit cell, (b) Front view, and (c) Side view

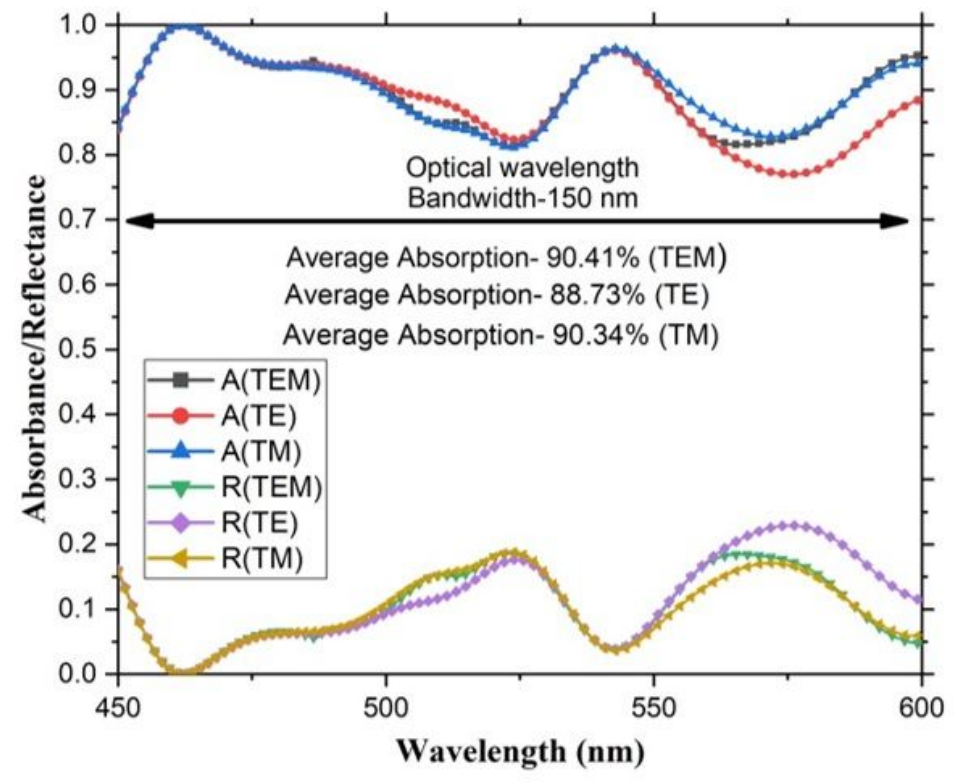

(a)

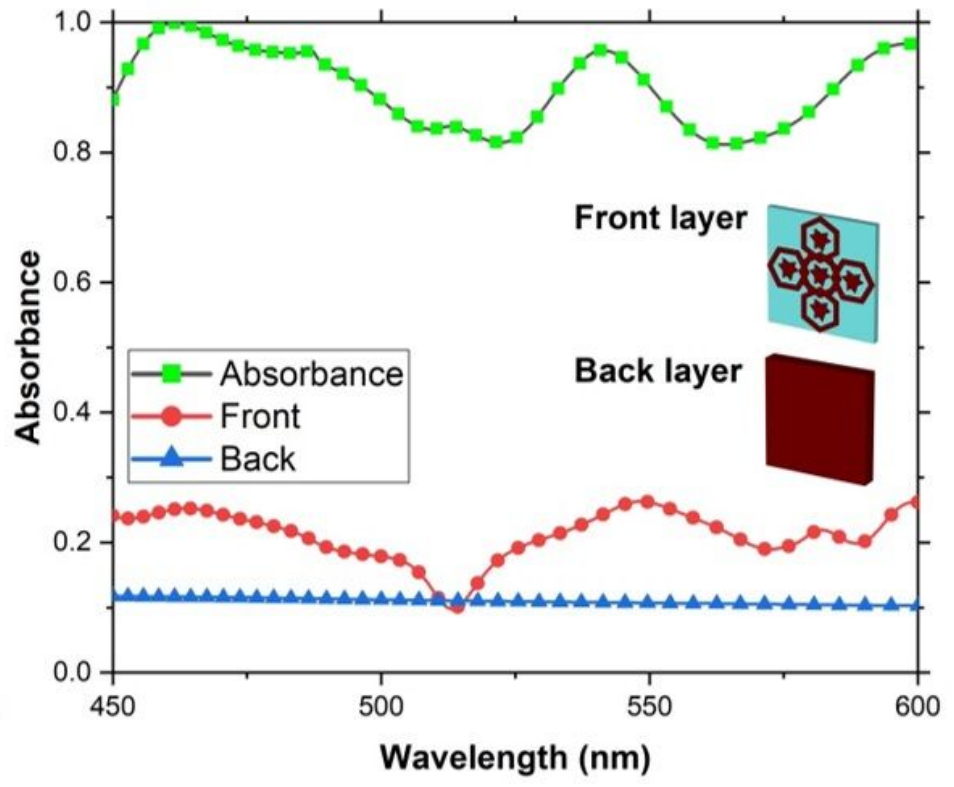

(b)

Figure 2

(a) Graphical representations of absorbance, reflectance, and transmittance for TE, and TM, TEM mode.

(b) Front and back-layer absorbance. 


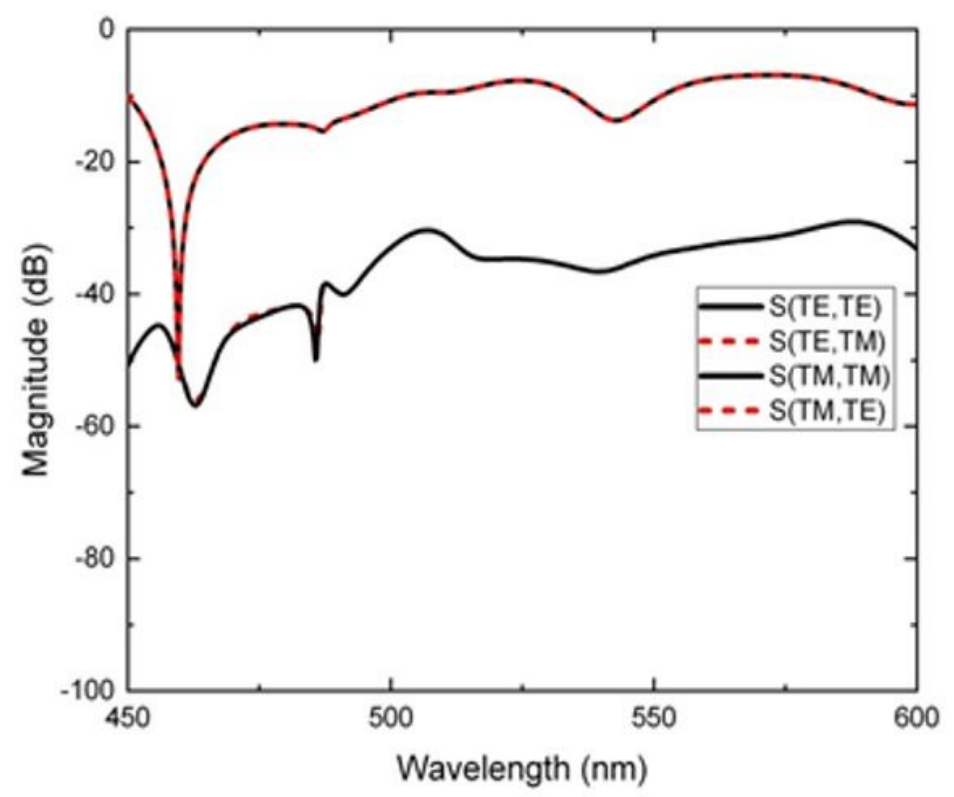

(a)

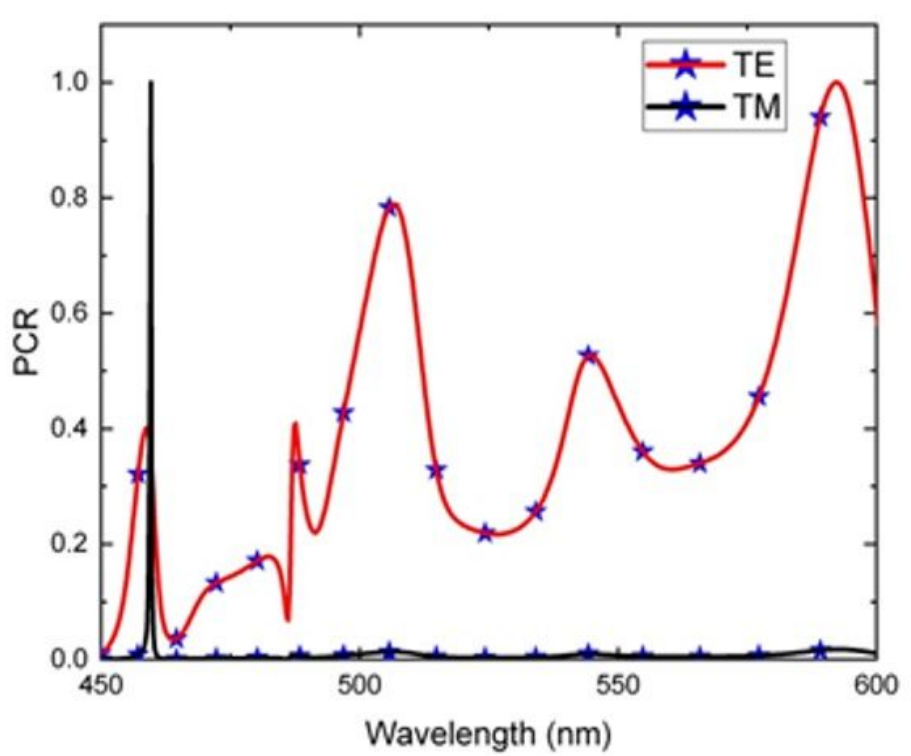

(b)

\section{Figure 3}

Co-polarization and cross-polarization magnitude (dB) for (a) Reflection coefficient (S11) for TE and TM modes (b) PCR for both TE and TM mode of optical region 


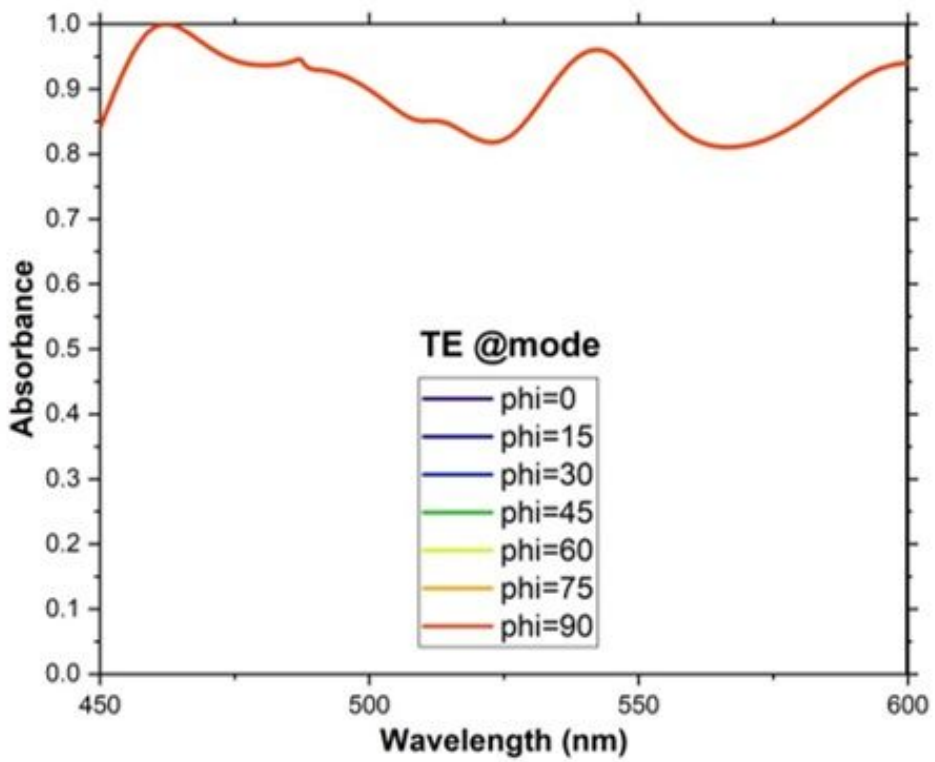

(a)

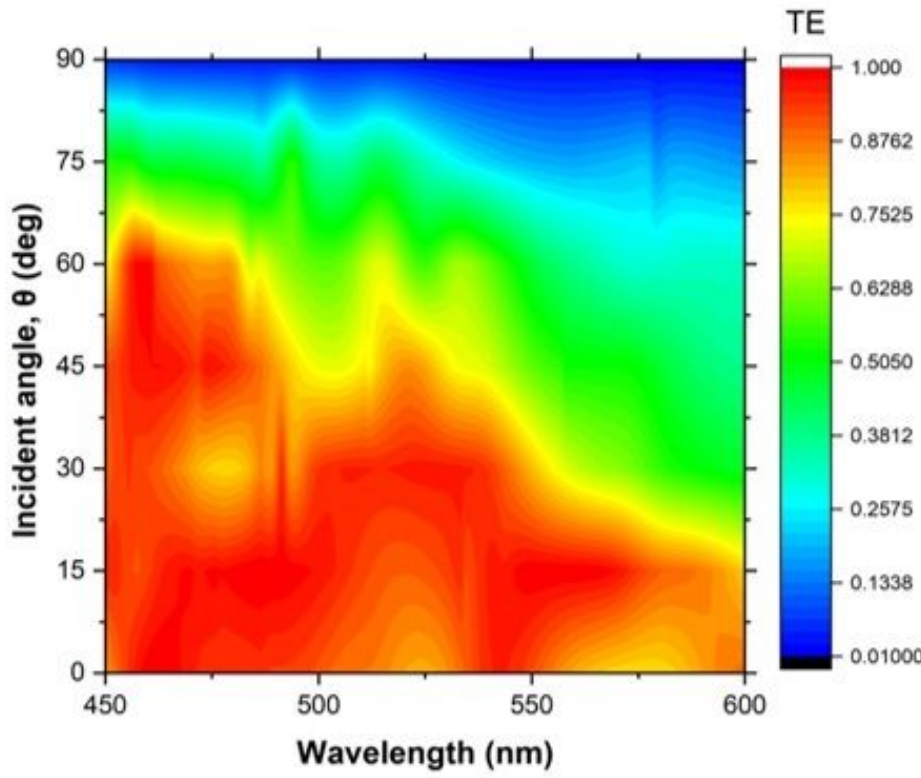

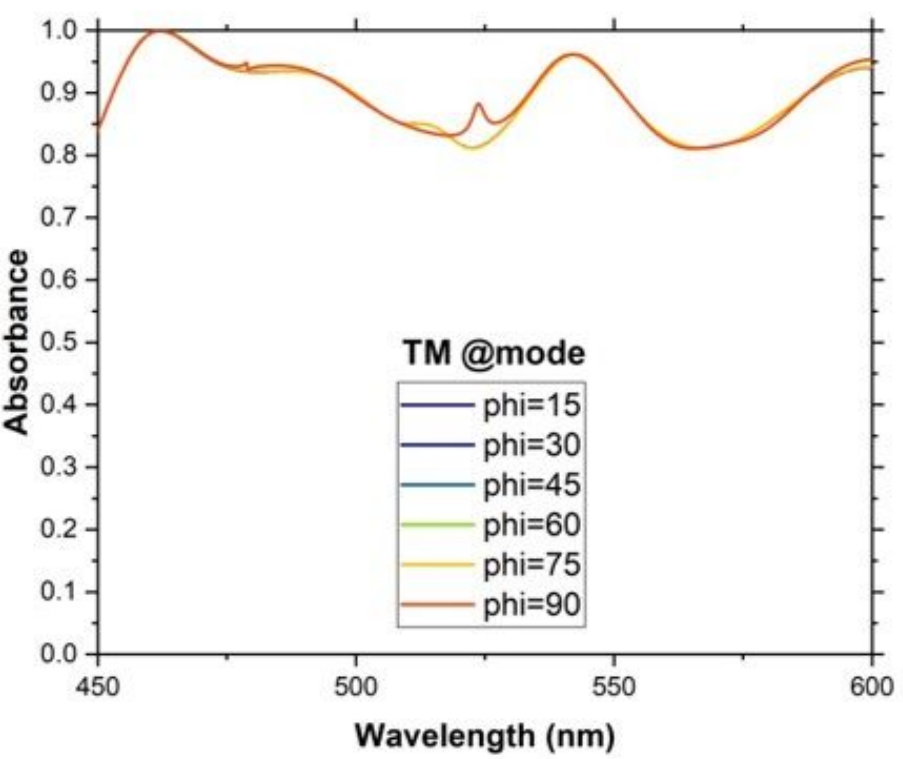

(b)

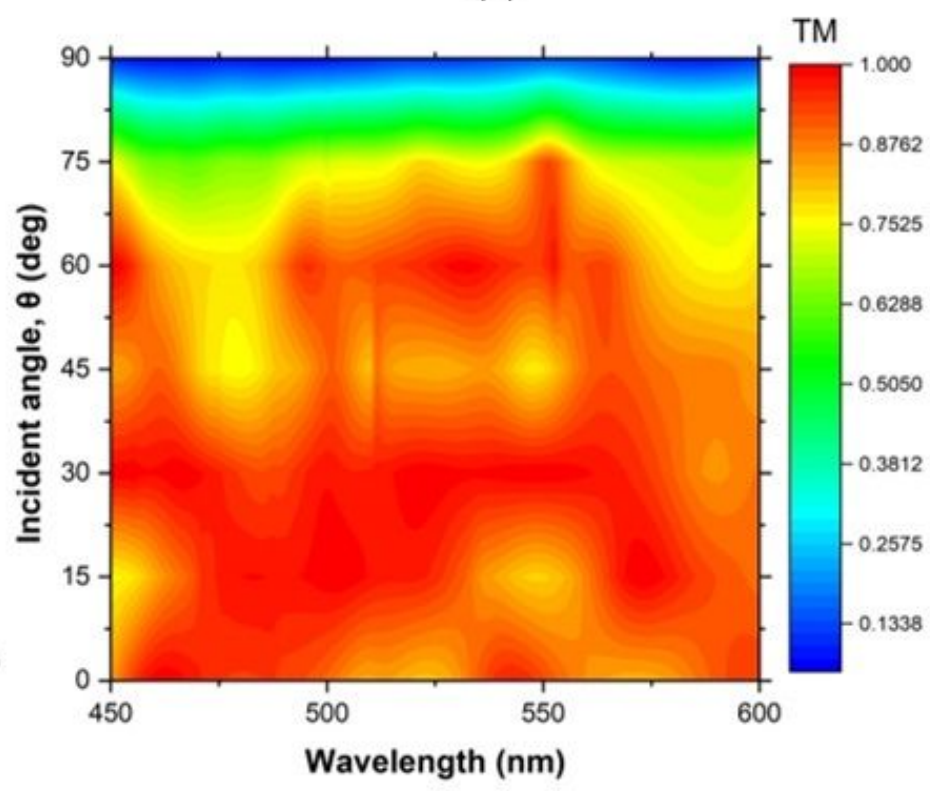

Figure 4

Absorption with distinctive polarization angle phi ( $\Phi(\mathrm{a})$ TE mode (b) TM mode and Absorption with distinctive incident angle theta $(\theta)$, (c) TE mode (d) TM mode. 


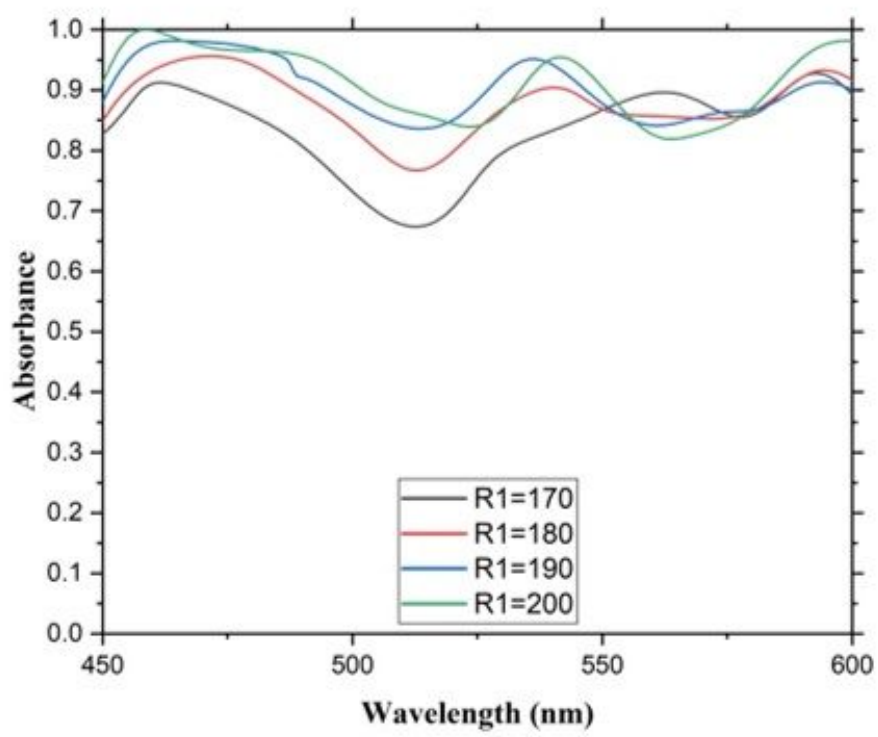

(a)

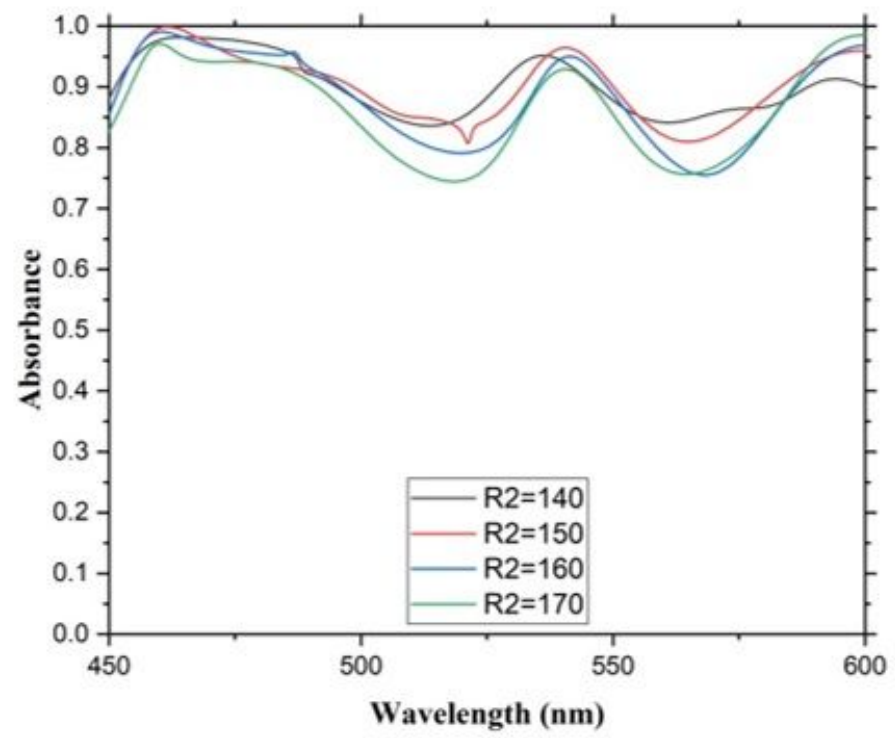

(b)

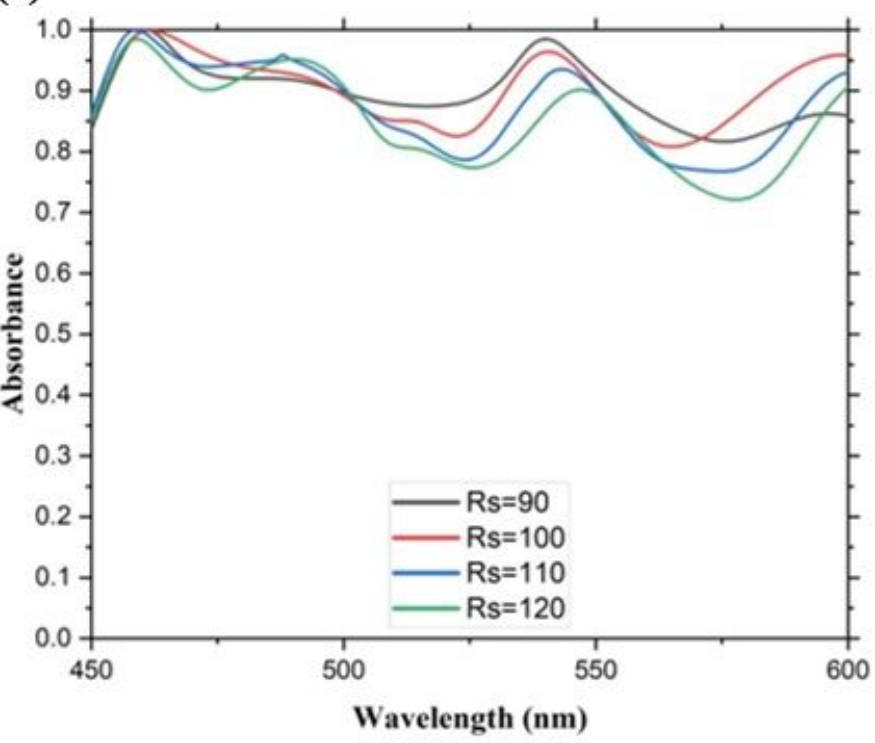

(c)

\section{Figure 5}

Wavelength response to a parameter sweep (a) Outer radius of hexagonal shape "R1", (b) Inner radius of hexagonal shape "R2" and (c) Radius of star shape "Rs" 


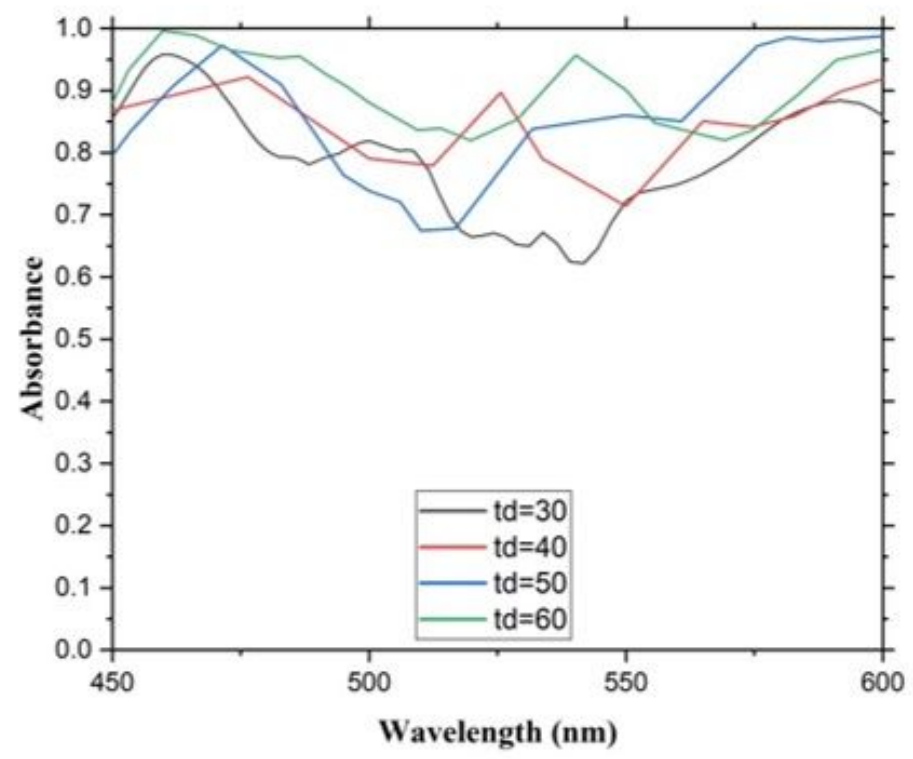

(a)

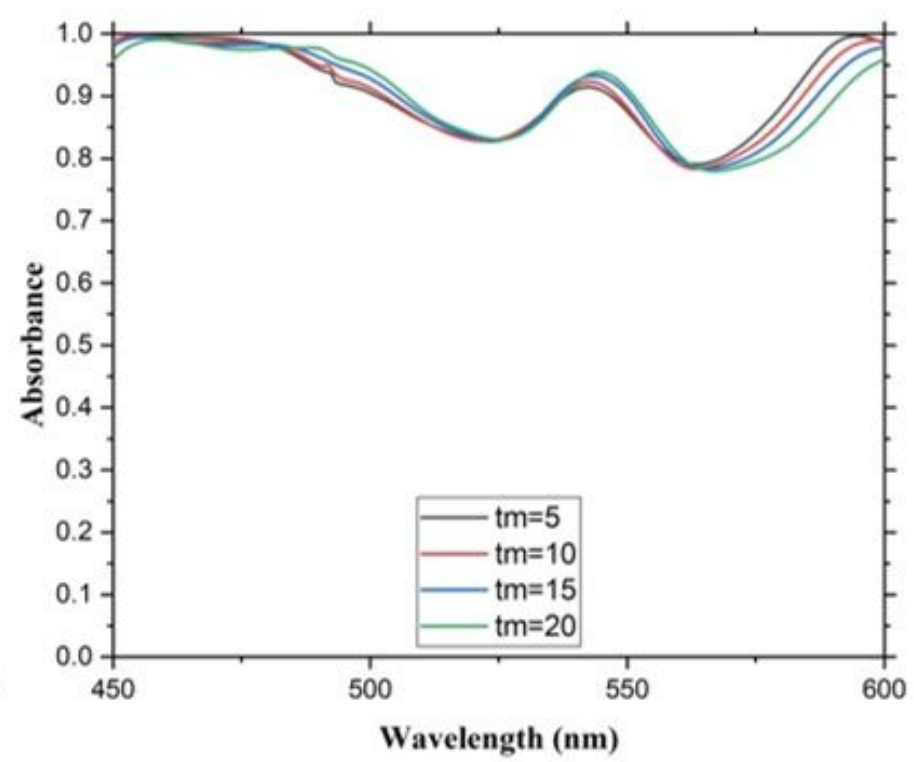

(b)

\section{Figure 6}

(a) Dielectric layer thickness "td" and (b) Resonator layer thickness "tm"

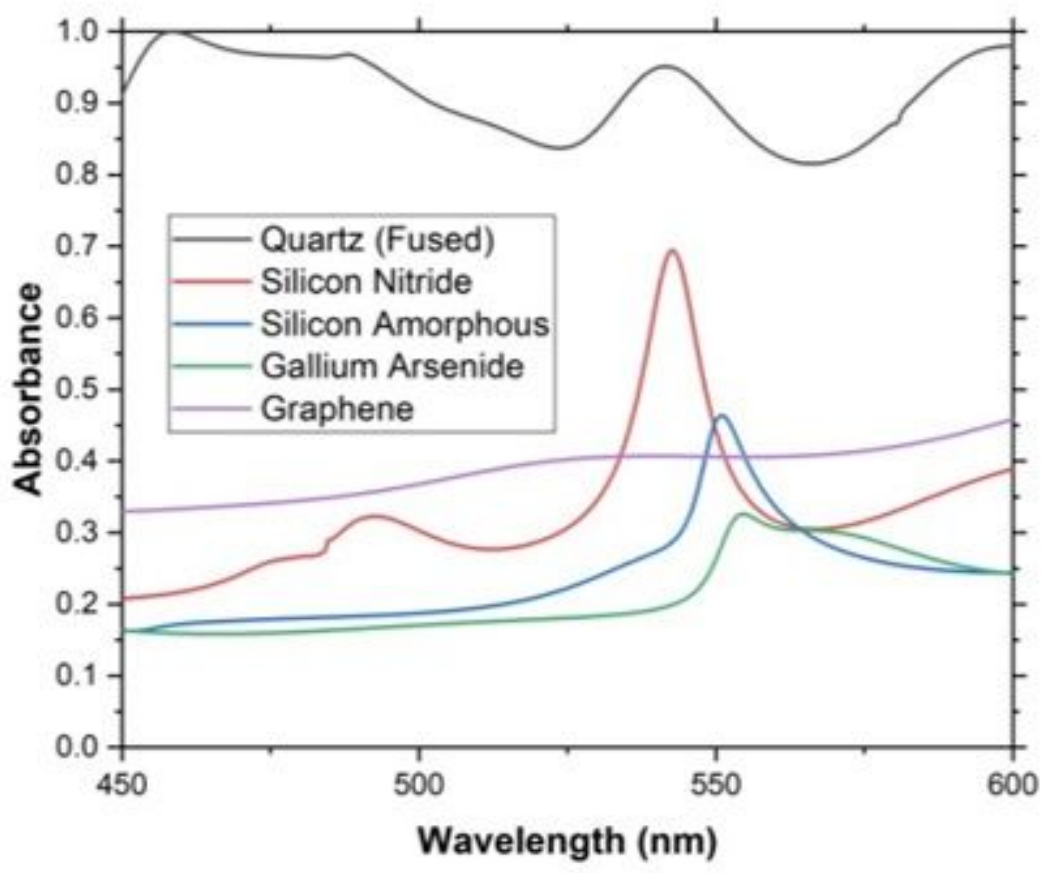

Figure 7

Different materials absorption analysis 


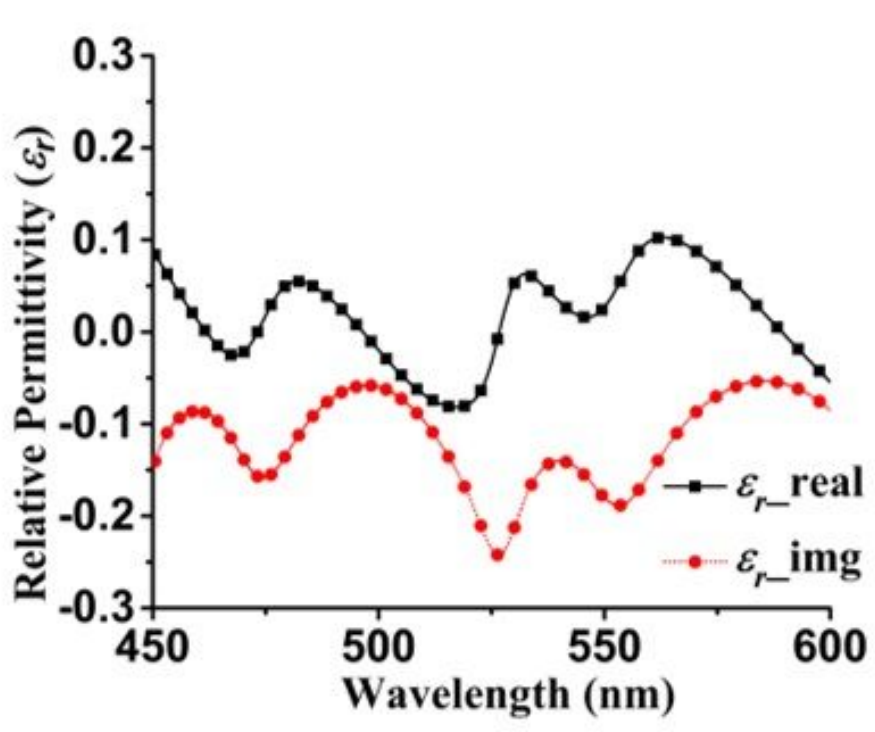

(a)

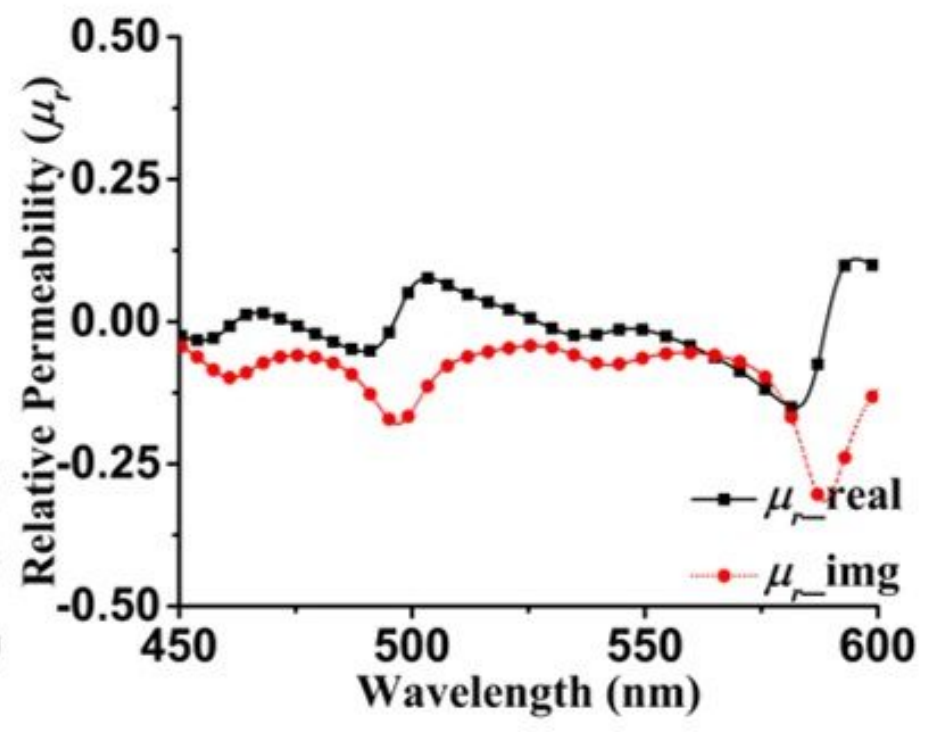

(b)

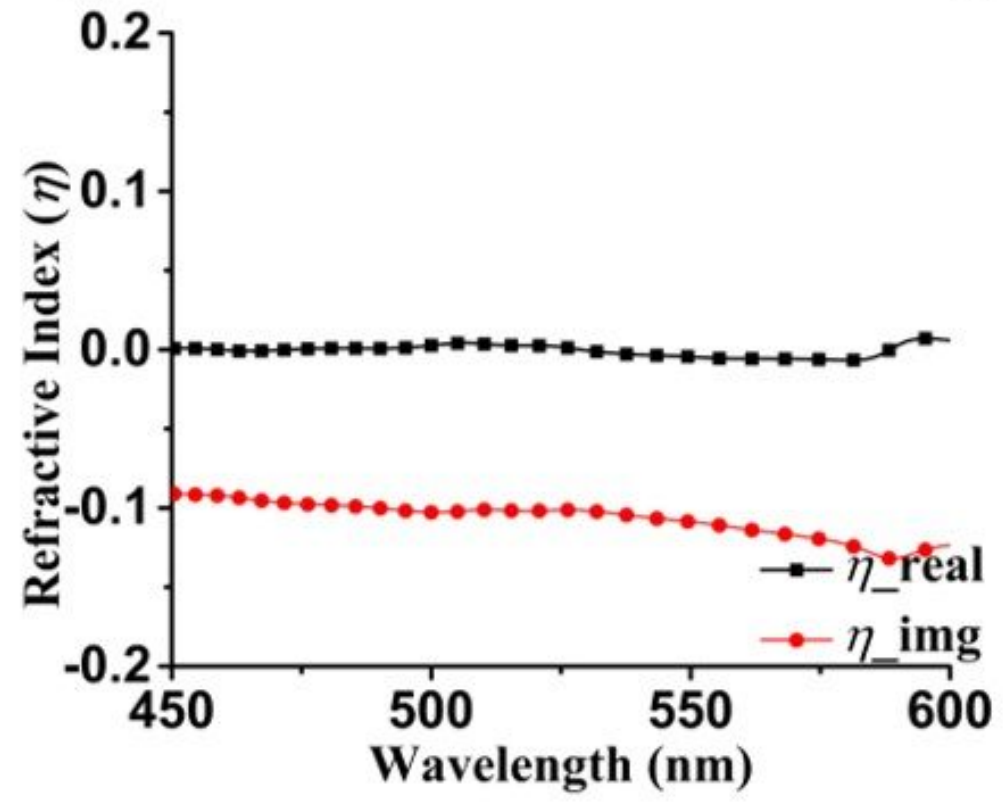

(c)

Figure 8

Proposed metamaterial absorber dielectric properties characterization (a) Relative Permittivity (b) Relative Permeability (c) Refractive Index 


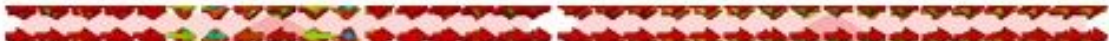

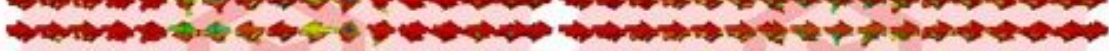

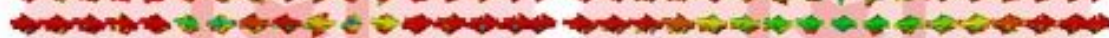

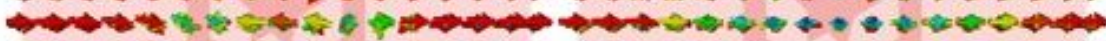

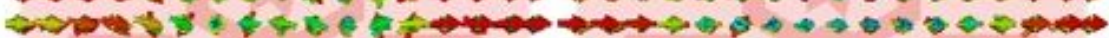

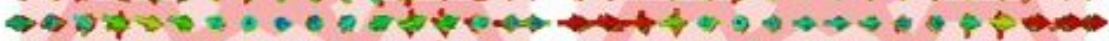

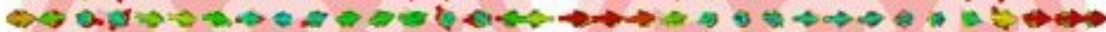

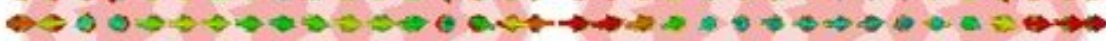

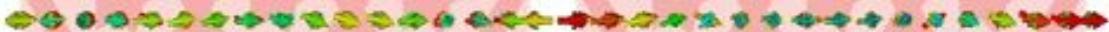

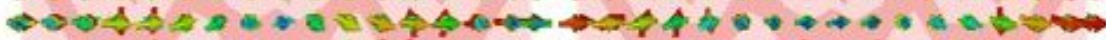

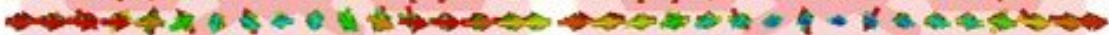

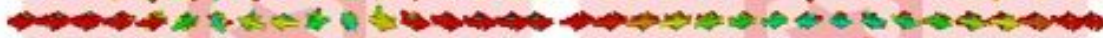

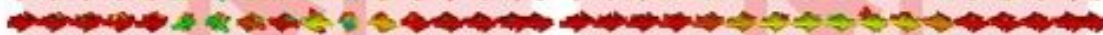

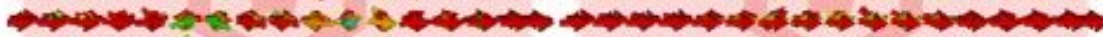

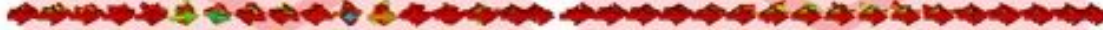

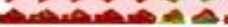

(a)

(b)

Q

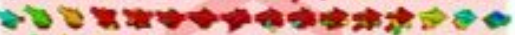

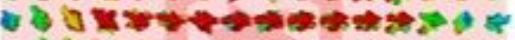

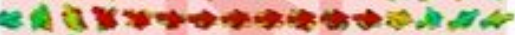

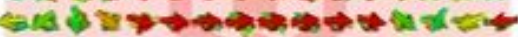

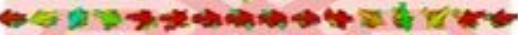
-

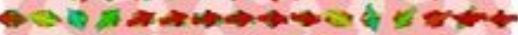

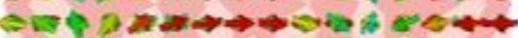
sif \&

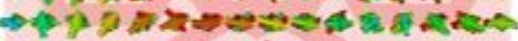

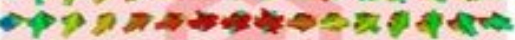

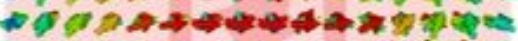
- 0 PAd

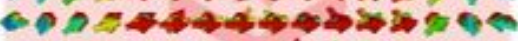

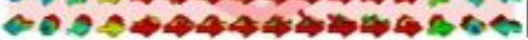

(c)

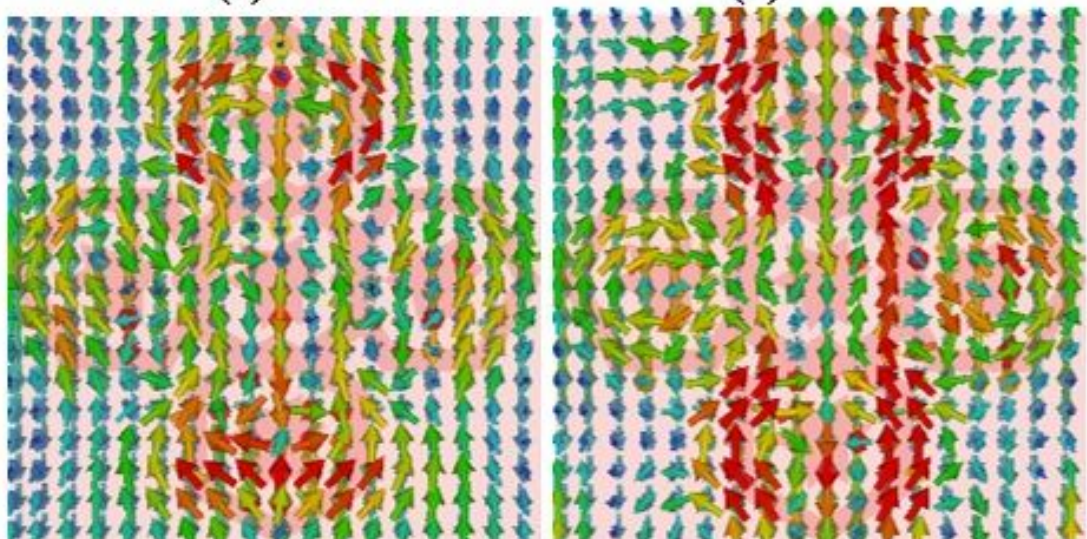

(d)

(e)

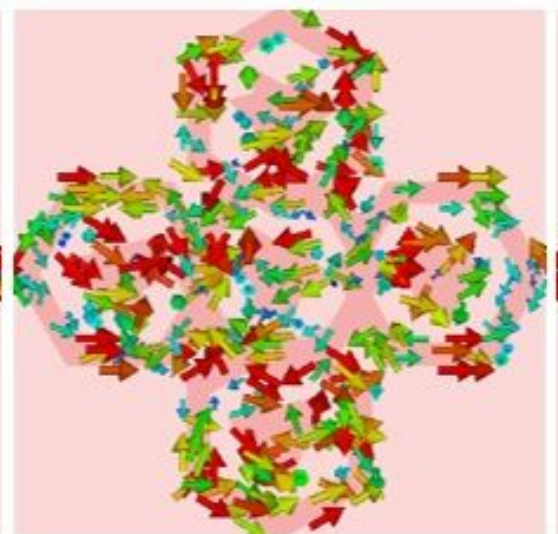

(h)

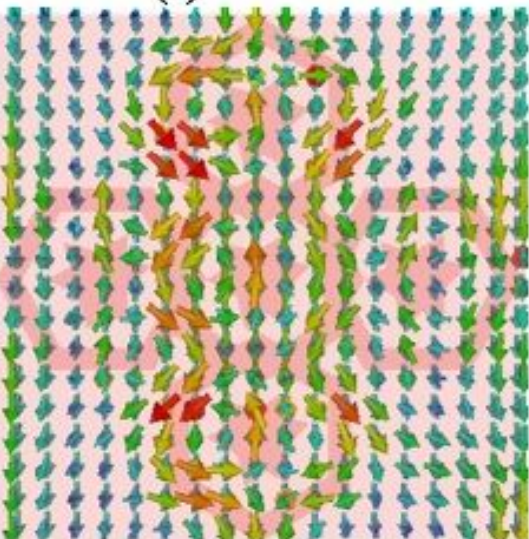

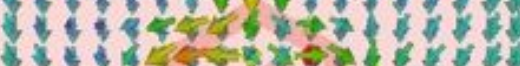

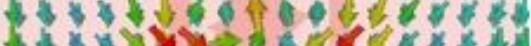

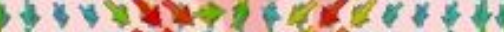

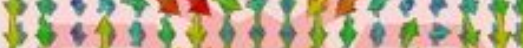
44 का की - 45 की

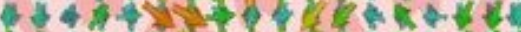

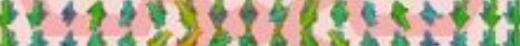

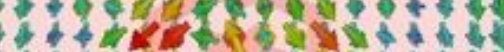

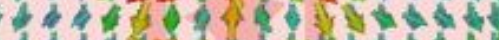

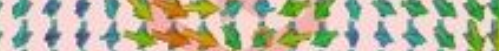

(f)

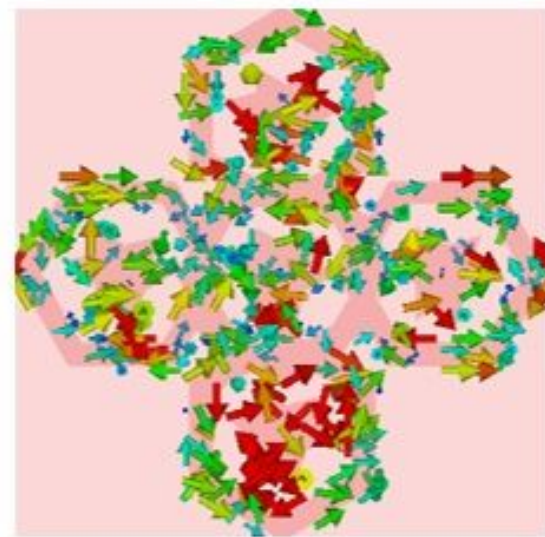

(g)

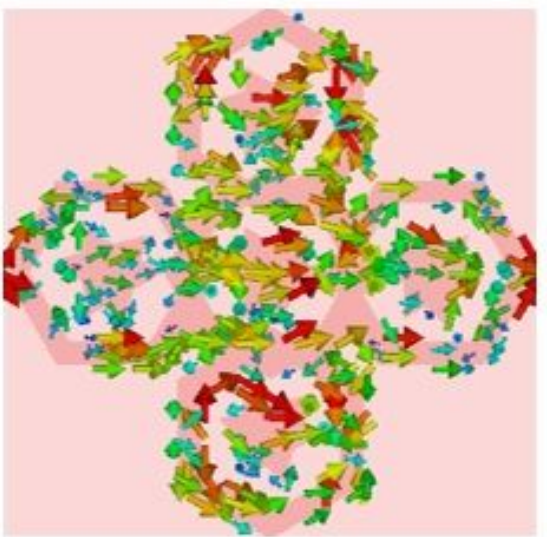

(i)
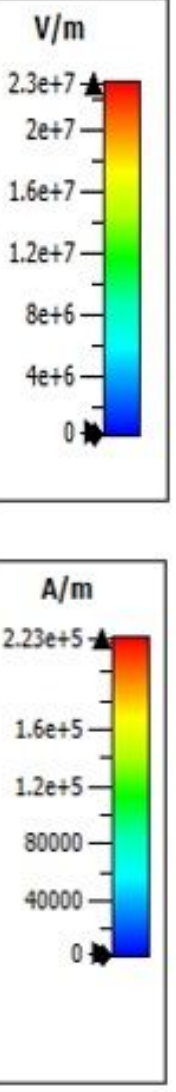

\section{Figure 9}

Surface current distribution of proposed MMA: a, b, c ; d, e, f ; g, h, l, respectively demonstrate $(E)$, (H) and surface current distribution of the resonator at $458.54 \mathrm{~nm}, 480 \mathrm{~nm}, 540 \mathrm{~nm}$. 


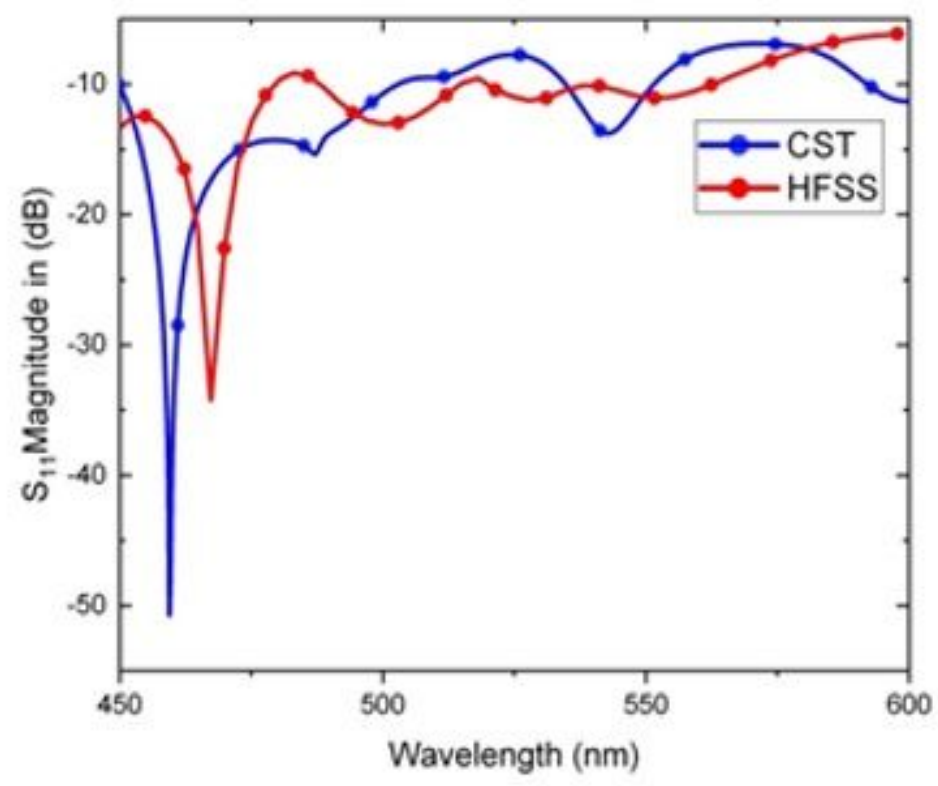

(a)

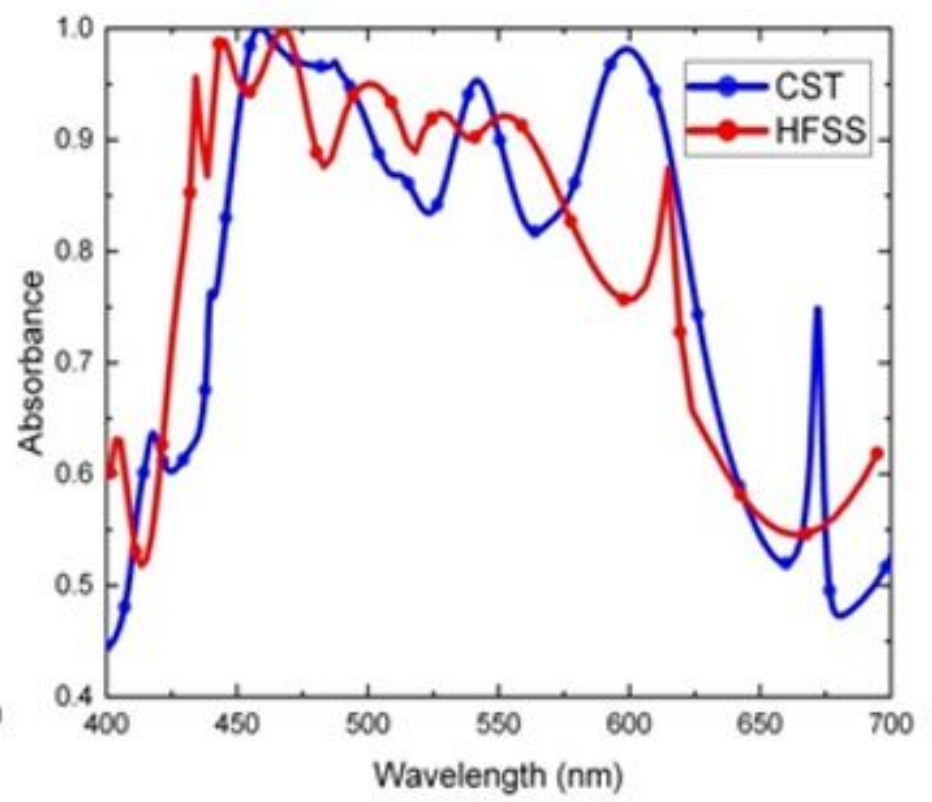

(b)

Figure 10

Comparison of different simulation methods with the S11 parameter (a) Magnitude in $\mathrm{dB}$ (b) Comparison of absorption with different simulation methods 